\title{
UNIVERSAL PAYLOAD INFORMATION MANAGEMENT
}

\author{
Ralph B. Elmore \\ Email: ralph.elmore@msfc.nasa.gov
}

\author{
Lockheed Martin Space Operations, Integrated Technology Solutions (ITS) \\ for the \\ National Aeronautics and Space Administration (NASA) \\ George C. Marshall Space Flight Center (MSFC) \\ Flight Projects Directorate \\ Ground Systems Department
}

\section{Introduction}

As the overall manager and integrator of International Space Station (ISS) science payloads, the Payload Operations Integration Center (POIC) at Marshall Space Flight Center has a critical need to provide an information management system for exchange and control of ISS payload files as well as to coordinate ISS payload related operational changes. The POIC's information management system has a fundamental requirement to provide secure operational access not only to users physically located at the POIC, but also to remote experimenters and International Partners physically located in different parts of the world. The Payload Information Management System (PIMS) is a ground-based electronic document configuration management and collaborative workflow system that was built to service the POIC's information management needs.

This paper discusses the application components that comprise the PIMS system, the challenges that influenced its design and architecture, and the selected technologies it employs. This paper will also touch on the advantages of the architecture, details of the user interface, and lessons learned along the way to a successful deployment. With PIMS, a sophisticated software solution has been built that is not only universally accessible for POIC customer's information management needs, but also universally adaptable in implementation and application as a generalized information management system.

\section{PIMS Applications and Capabilities}

PIMS consists of three main applications that are part of the POIC's arsenal of Web based applications. The "Documents" application is an electronic document configuration management system used by the POIC as a controlled repository of payload operations files. The "Operations Change Request" (OCR) application is a workflow system for managing and approving changes needed during real time payload operations. The "To Do List" application is similar to an email client and provides access to user specific collections of current OCR and Document tasks requiring action. Further details of these applications are explored in the paragraphs that follow.

\section{Documents}

The Documents application provides consolidated access to a central repository of the approved versions of documents and files for a specific mission. The appearance of the Documents application is similar to graphical file system traversal application, with the documents and files being organized under folders (see Figure 1 - Documents Application Main Window). A notable difference is that files reside in a document container rather than directly under a folder. A document container may hold any number of 
files. It is also used to associate additional metadata, such as a title, description, and an email notification list. The document container is the object under primary configuration management control (i.e. if a file change is desired, a revision is performed on the document to change the file). Multiple versions of documents and files are maintained, with checkout and check-in features supported for revision.

Document and file revisions are performed in isolation on an in-work copy of the document. The in-work copy is manipulated from the user's To Do List. A document revision will not be publicly accessible until published by the editing user. When new versions become available, the system will automatically generate email notifications of version availability to a document specific list of designated addresses.

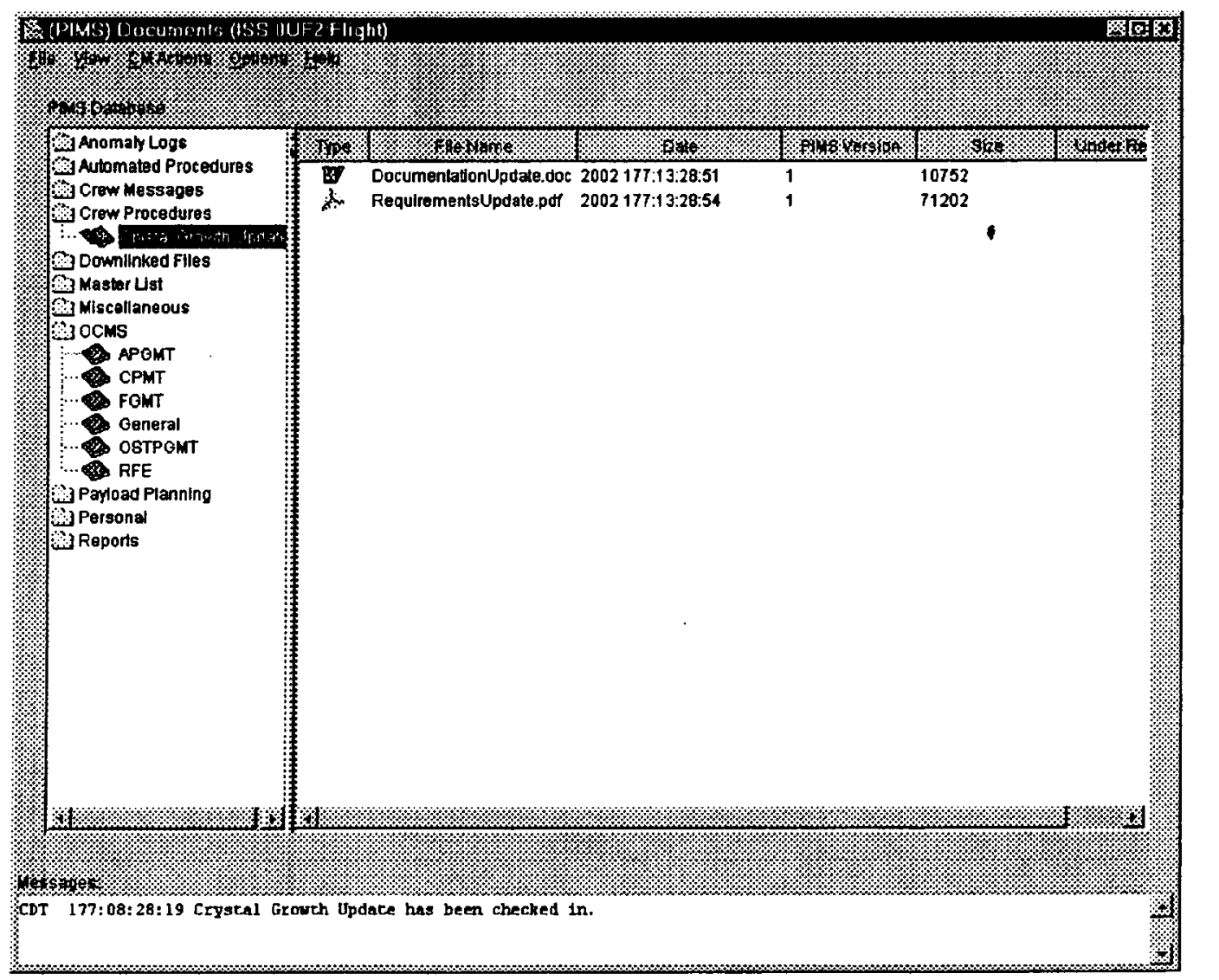

Figure 1 - Documents Application Main Window

The Documents application is capable of transferring files between the PIMS repository and the machine running the PIMS client (for import, export and integrated viewing), as well any machine running an FTP server (import and export only). The Documents application is also used to transfer files from the PIMS repository to a Johnson Space Center (JSC) accessible drop-box for spacecraft uplink. A background polling process also monitors external facility (e.g. JSC) drop-boxes and will automatically retrieve designated files and place them in the PIMS repository.

There are also legacy POIC applications that require access to PIMS services. An API library interface exists to support programmatic storage and retrieval of files as well as access to document and file information.

Other features built into the Documents application for operating on the PIMS repository include capabilities to compare files, perform a byte swap of files, perform a string search against stored files, and the ability to bulk import files, folders, and documents from an object definition file. 


\section{OCRs}

The OCR application provides a collaborative, electronic change request system for coordinating the approval and implementation of real time changes desired for spacecraft payload operations. The OCR application provides the capability to create, view, manipulate, and query information concerning OCRs (see Figure 2 - OCR Application Main Window). The application's real-time status displays are maintained with dynamic display updates. The OCR application encapsulates a configurable workflow process which routes OCRs through an entire life cycle of development, approval, implementation, and archival. All OCR workflow processes are coordinated through and routed to individual users via the "To Do List" application.

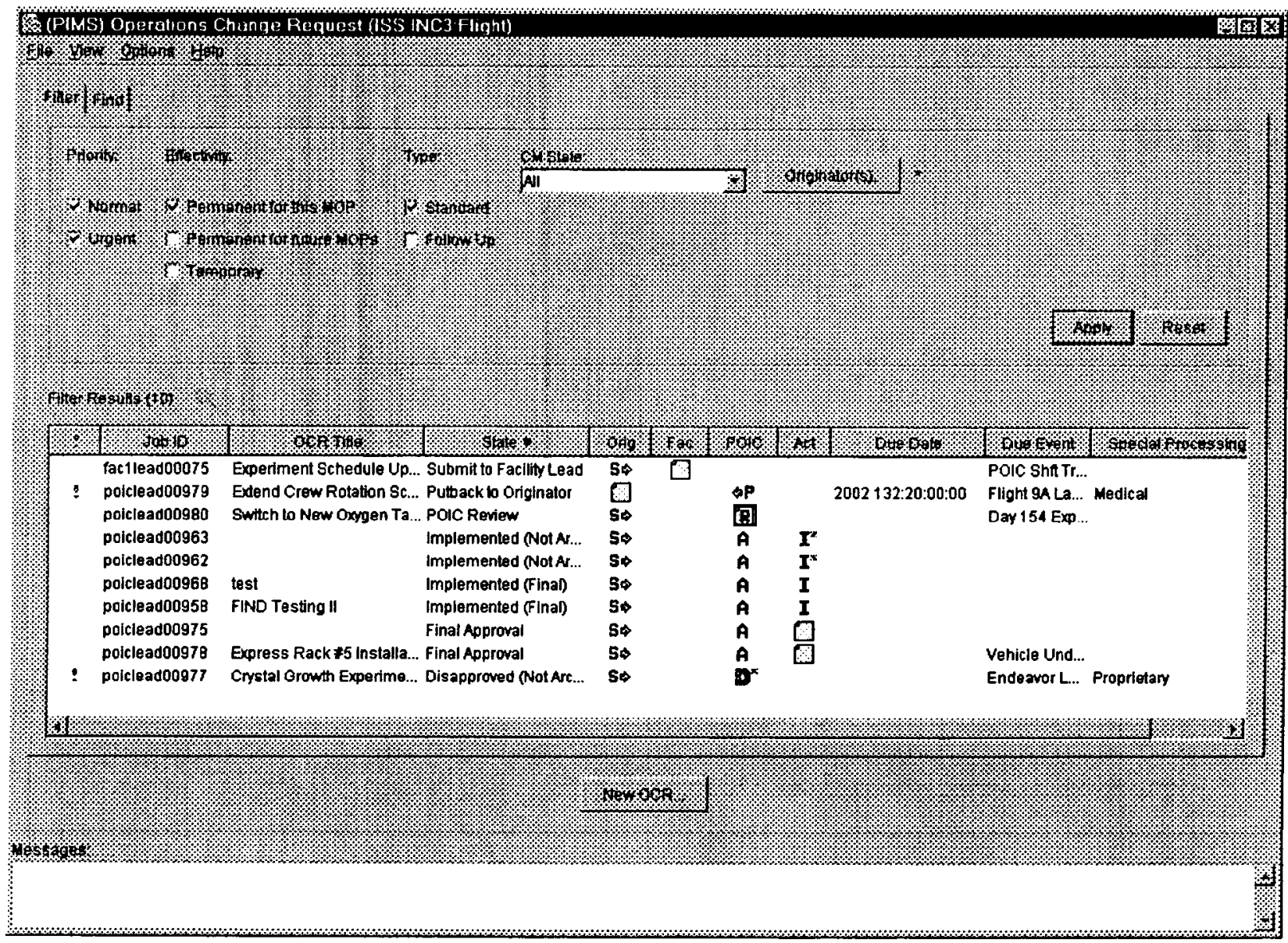

Figure 2 - OCR Application Main Window

The underlying workflow that supports operations change requests is a mix of both fixed and dynamic steps. In its simplest form, an operations change request starts with an originator, is approved by a lead, and implemented by a capable individual. As required and depending on the change, an OCR approval can be much more involved. It can be routed through hierarchies of approval with optional review phases at each tier of the hierarchy. Reviewers can coordinate subordinate reviews with team members or other individuals within an area of expertise. Likewise, an OCR implementation can be constructed with complex implementation sequences consisting of sequential, parallel, hierarchical, and independent task actions. Refer to Figure 3 - OCR Workflow Diagram for complete details of the OCR workflow process. 


\section{PIMS OCR Workflow}

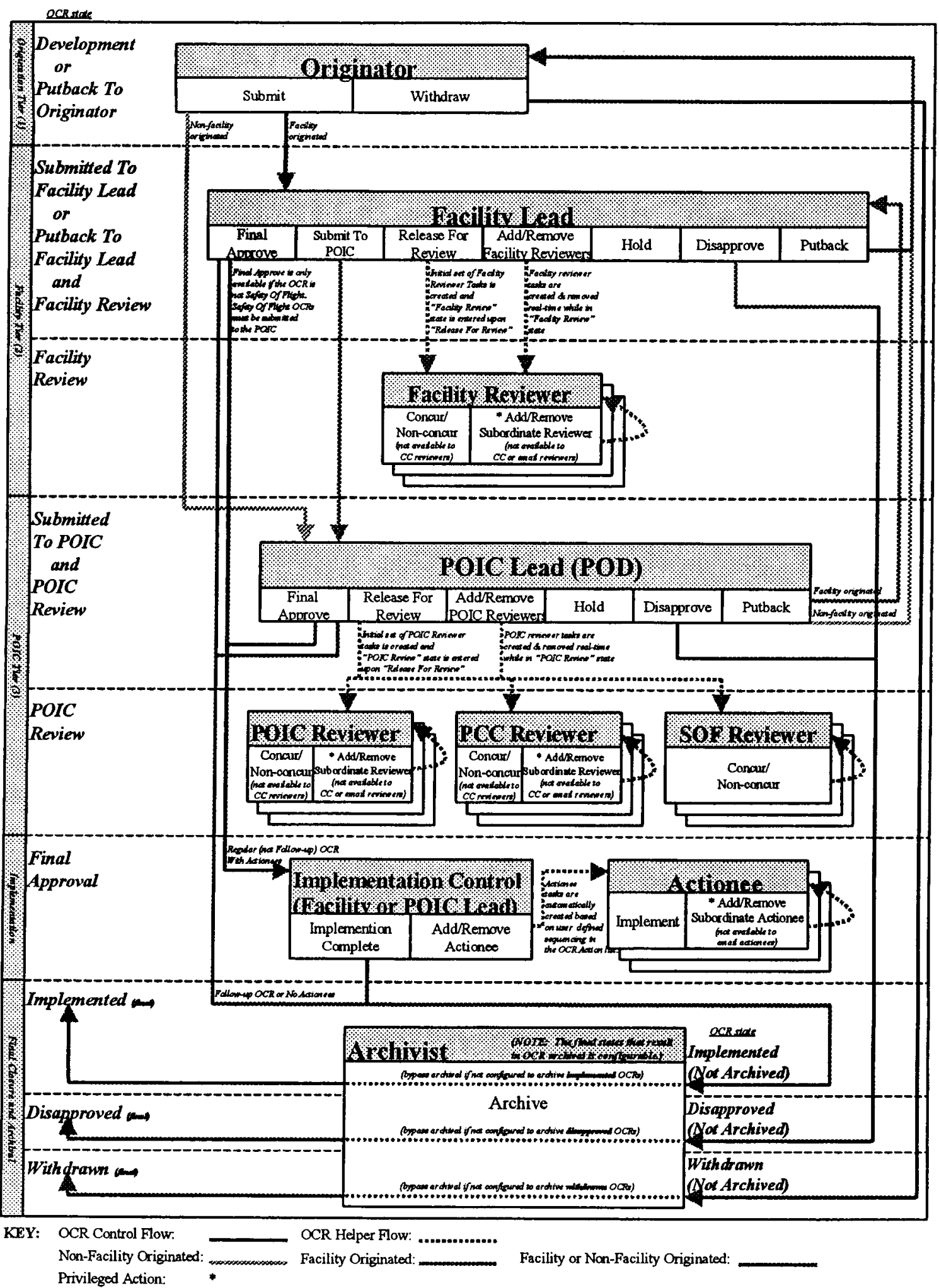

Figure 3 - OCR Workflow Diagram 
To further illustrate the OCR workflow, the following flow diagram (Figure 4 - Sample OCR Workflow) is a hypothetical flow of a non-facility originated OCR (e.g. it was originated by a POIC member) that was routed through the entire workflow process. This OCR was:

[1] submitted to the POIC lead,

[2] released for POIC review,

[3] sent on by one POIC reviewer for subordinate review to two additional reviewers,

[4] subsequently approved by the POIC lead,

[5] implemented with a root sequence of 2 parallel steps (labeled 1) followed by 2 sequential steps (labeled 2 and 3),

[6] as part of the implementation of step 2, was routed through 2 subordinate sequential steps (labeled 2.1 and 2.2),

[7] implementation was then declared complete by the POIC lead, and finally

[8] archived to fully complete the OCR life cycle.

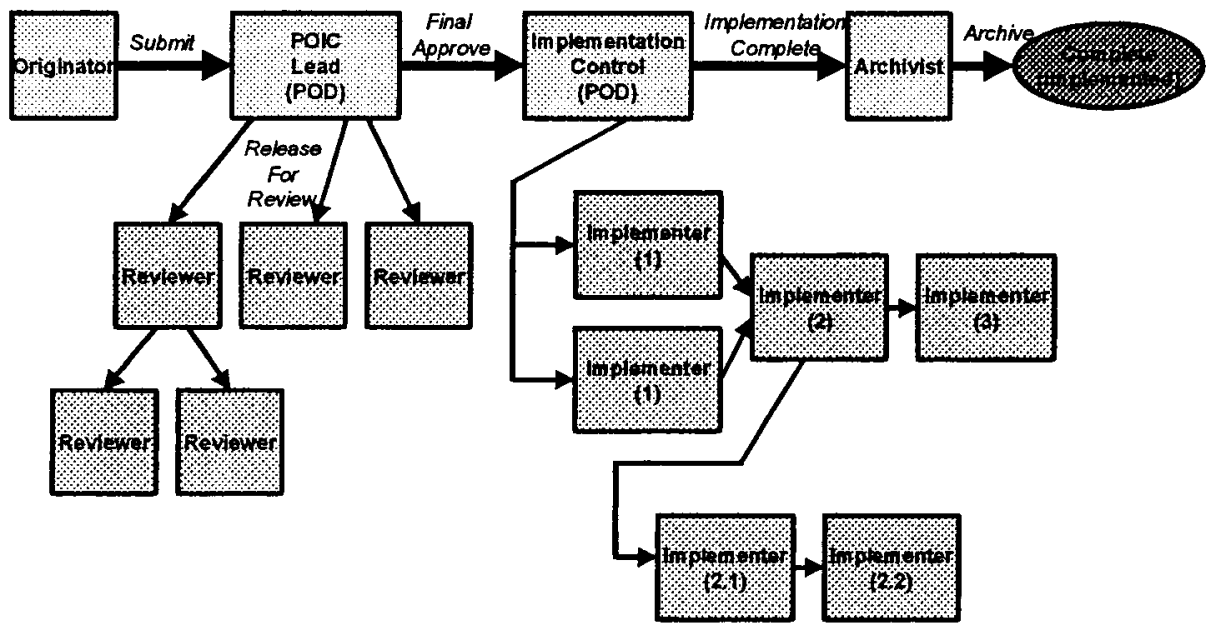

Figure 4 - Sample OCR Workflow

While an OCR is in process, the capability exists for users to store supporting and reviewer attachments of any file type to an OCR. Email messages can be sent to users with notification of OCR progress through the development, approval, and implementation process. Certain actions, such as OCR review or action implementation by non-PIMS users, can be coordinated through automated, system-generated email messages. Lastly, each user involved in the OCR workflow has a log area in which they can permanently record any information concerning their task implementation as part of the OCR.

\section{To Do List}

As previously stated, the "To Do List" is an email-like application that provides a list from which users view and operate on current workflow tasks they are required to perform relating to the approval of a Document or an OCR (see Figure 5 - To Do List Application Main Window). The "To Do List" task display is dynamic. Tasks can be automatically added or removed by workflow actions at any time. "Complete Task" screens brought up from the "To Do List" focus the user on the action required and restrict the user's actions to only what is appropriate for the task. Surrogate tasks can involve non-PIMS users in an OCR review or its implementation utilizing email. 


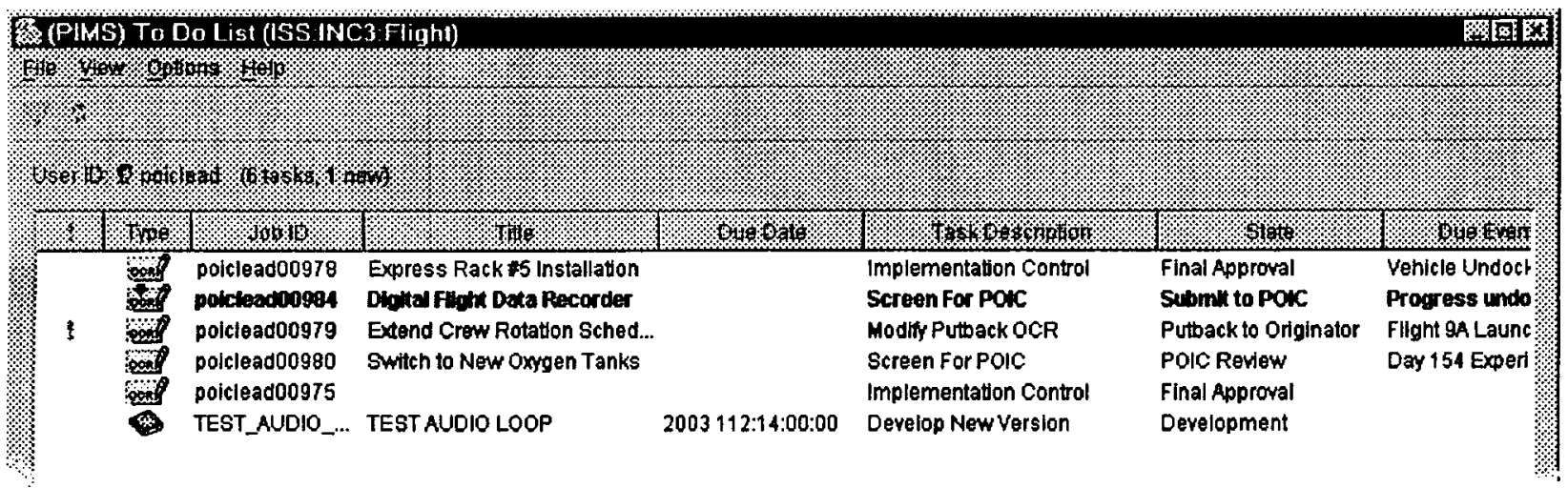

Figure 5 - To Do List Application Main Window (partial)

\section{Design and Architecture}

\section{Challenges}

Typical application development challenges existed with the PIMS system. These included industry common issues such as operations concept, external interface, requirement, and technology flux. PIMS also had varying degrees of involvement from the user community. Early in the process there was limited user involvement, with some categories of "power" users being excluded altogether. Beyond these common issues, there were notable application specific challenges in the areas of accessibility, security, and user interface needs.

The heart of PIMS is its centralized storage facility that provides full transaction support to ensure data integrity. The POIC must provide secure access to the centralized PIMS repository and related services to its entire POIC user base. The user base includes individuals physically located at the POIC as well as remote users and facilities. Some users have dedicated connections, while others must access PIMS over the Internet. As such, a controlled access web-based user interface is a fundamental requirement of the system and a major challenge.

Another challenge centered on the Graphical User Interface (GUI) requirements of the PIMS system. It is expected to have the look and feel, input validation, and relative response of a native application (reference previous examples are included in this paper: Figure 1- Documents Application Main Window, Figure 2 - OCR Application Main Window, and Figure 5 - To Do List Application Main Window). The PIMS GUI is expected to function similar to existing POIC developed applications and commonly used COTS products. Further complicating the GUI are requirements to enforce data interrelationships and input validations real time, with each mouse-click and keystroke. It was therefore not possible to rely on traditional, HTML based, form submittal and validation. Additional GUI challenges are desires for it to function similarly on multiple platforms and for it to process real time updates such that the graphical information display always shows current information.

The PIMS system, while being engineered for web accessibility, also has requirements to interface with legacy systems written primarily in C. These legacy systems required an API library interface that could be linked into the legacy application executable. 
The PIMS system is required to support many file transfer interfaces. Among the possible components exchanging of files with the centralized PIMS storage mechanism are:

1. PIMS Web GUI client user workstations.

2. Any FTP server controlled by a PIMS Web GUI client. This is known as a 3-point transfer.

3. Legacy applications, through the aforementioned API library interface.

4. The POIC drop-box, for file exchange with interfacing facilities.

5. The PIMS POIC drop-box polling program, which pulls and imports files from POIC interfacing facilities.

Other design challenges included automated system emails for both status notification and workflow involvement and PDF report generation capabilities.

\section{Selected Technologies}

In order to address these outlined design challenges, several modern architectural programming technologies were used.

Java was selected as the primary programming language. It is portable, allowing for platform independence, and web friendly, as it can be run from a browser. The swing component library of Java allows the creation of the required sophisticated GUI. Additionally, enterprise level component libraries exist for Java to support database interaction, email, messaging, and XML parsing.

A Structured Query Language (SQL) based database with ACID (Atomicity, Consistency, Isolation, and Durability) compliance was chosen as the central repository. ACID compliance ensures transaction and data integrity. SQL compliance allows the use of the Java Database Connectivity (JDBC) interface for access from Java. In addition, a SQL based database accessed through a standard JDBC programming interface allows great flexibility in choosing or switching database vendors.

Common Object Request Broker Architecture (CORBA) was selected as the distributed object backbone of the PIMS architecture. The CORBA ability to pass complex objects between systems satisfies tier-totier communication needs. The inter-language capability of CORBA by using Interface Definition Language (IDL) facilitated the integration of legacy software and applications.

For security, several technologies were chosen. These technologies are part of the overall POIC web infrastructure of which PIMS is a component and not directly built into the PIMS software. A Virtual Private Network (VPN) is utilized for network security. The VPN provides user and host based authentication. In addition, the encryption capabilities on the VPN "pipe" eliminate encryption needs for individual application connection mechanisms. Object signing allows certification of the client software. Common web security measures such as the use of HTTPS, certificates, password authentication, and account privileges provide additional layers of security.

Finally, as a general rule, an effort was made to ensure that PIMS utilized ubiquitous technologies and file formats based on standards. Sticking with standards makes the system adaptable and allows it to easily interface with other products. Some examples include the use of an integrated File Transfer Protocol (FTP) client for exchanging files between the PIMS repository and non-PIMS systems. Simple Mail Transfer Protocol (SMTP) is used for all PIMS generated emails. Extensible Markup Language (XML) is used for object import into the "Documents" application. Portable Document Format (PDF) is used as the format of all generated reports. Lastly, Zip format is used for archives generated through the OCR archival process. 
The following diagram (Figure 6 - PIMS Architecture Diagram) illustrates the platform and component architecture of the PIMS system.

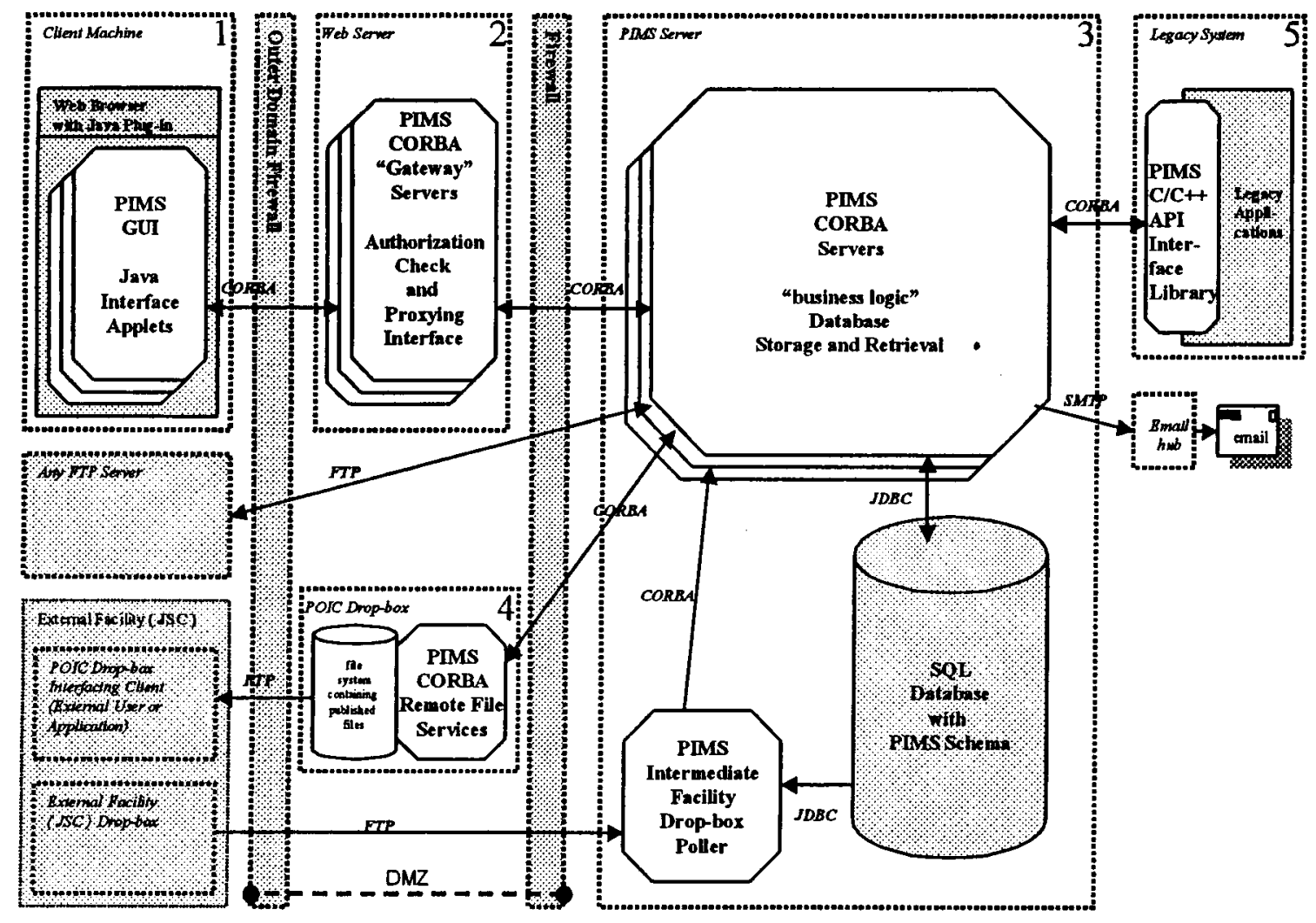

Figure 6 - PIMS Architecture Diagram

As can be seen in this diagram (Figure 6 - PIMS Architecture Diagram), PIMS employs a multi-tiered web-based architecture with a centralized SQL database accessed via Java JDBC drivers. Separate GUI (diagram box 1), gateway (diagram box 2), and business logic layers (diagram box 3 ) have the advantage of separating the presentation from the implementation. Even though "thin clients" (strictly a served GUI with limited front-end logic) are not used, this multi-tiered architecture still has many the advantages of a thin client system. Business logic can be changed without impacting the clients.

PIMS client applications (diagram box 1) run as Java applets hosted by a Web browser and are part of a larger POIC web application suite. The Java virtual machine is provided by the required Java plug-in. All code runs from pre-installed, signed Java JAR files. Client users maintain persistent connections to the web server. However, all service requests are transaction oriented. Hosting in the Web browser also provides a key PIMS feature, file viewing. Integrated file viewing is accomplished by instructing the browser to open local URLs, thus invoking the native viewing application.

The gateway tier (diagram box 2 ) is primarily a security layer. The gateway is located in a controlled demilitarized zone (DMZ) network area. The PIMS gateway tier software further enhances security by 
authenticating user requests on a transactional basis and by proxying authenticated traffic to the secured business logic layer.

The heart of the system is the PIMS Server or business logic tier (diagram box 3). It implements nearly all application specific control logic. It also provides all interfaces with the SQL database.

Additional tiers include the legacy application PIMS API interface library (diagram box 5) and the POIC drop-box (diagram box 4) mechanism used to export files for retrieval by other facilities. Additionally, the diagram shows the drop-box polling program used to automatically import files and information from the drop-boxes of external facilities into PIMS.

\section{Architectural Advantages}

Numerous architectural advantages have already discussed in Selected Technologies and Platform and Component Architecture. However, it is worthwhile to both point out and re-iterate several key features:

1. This PIMS architecture is modular, scalable and highly distributable. It can be run on one box or distributed across many. Running multiple instances of CORBA servers can increase throughput.

2. The PIMS architecture is not platform dependent. Pervasive use of Java allows easy porting.

3. The PIMS architecture is adaptable. Use of pervasive, standards-based technologies allow easier integration into other environments and internal implementation swapping.

4. The PIMS architecture, as deployed at the POIC, is secure.

\section{Lessons Learned}

Over the course of developing the PIMS system, several important lessons were learned. First, iterative development was absolutely essential to the success of the system. With the challenges of requirements, concepts, interface, and technology flux, it was imperative to get capability to the users early so that they can begin the discovery and re-evaluation process while additional capability is being added. Further, it provides opportunities to recover from poor architectural component decisions and software implementations. Iterations provide necessary opportunities to continuously enhance and even re-invent the product to best meet the needs of both users and developers.

It is critical that end users, including those who are not necessarily driving the requirements, have direct communication with developers throughout the development process. An open communication channel keeps the users informed and contributing to the design. Users can contribute to the priorities of the next round of enhancements. End user communication also facilitates user satisfaction as they have contributed to the design throughout the iterative process. Minor software changes can often provide a major user savings in terms of time and satisfaction. An open developer-to-user dialog and user community surveys help identify both these minor changes with large user enhancement. It also helps to identify missed requirements and engineering opportunities that could further enhance a user's ability to perform their job.

It is important for developers to constantly monitor and evaluate new technologies. An emerging technology may suggest new and better solutions than originally planned, significant cost savings possibilities, or simply directions to steer the product on the next iteration.

By sticking with ubiquitous technologies based on standards, the system has maximum flexibility, adaptability, and acceptance. This approach makes it easy to replace costly or poorly performing implementations with alternatives. It also provides better interoperability with external COTS products and the desktop environments of a disparate user community. 
Infrastructure and utility level "Off-the-shelf" (OTS) components proved more useful and viable than adaptation of large scale, generalized "all-in-one" products that tend to dictate business logic. Early on, it was recognized the POIC needed a "document configuration management and workflow" system. There are commercial product suites that generically perform these functions. Initial incremental design and deployments attempted to tailor and integrate one of these large-scale proprietary products. This resulted in several problems:

- Generally speaking, the required integration code and the resultant system architecture overly complex.

- Limitations of the software prevented evolution of the integrated solution to better meet changing customer needs.

- Startup and recurring maintenance costs were high.

- Performance could not be optimized to a level acceptable for real time operational use.

- Fragmentation of the system components occasionally compromised data integrity on failures.

Ultimately, large-scale "all-in-one" products were abandoned for PIMS. Their use is not advised unless operations concepts, processes, requirements, and user interface needs can be easily adapted to conform to the selected tool. Integration of infrastructure and utility OTS components that had no influence on business logic or requirements were more rewarding for the PIMS system. Examples of these types of products include implementations for SQL database, CORBA, task specific libraries (XML parsing, email, PDF report generation, FTP, etc.), and virus scanning.

Even good selection of OTS components comes with a potentially overlooked maintenance issue. The interrelationships of upgrading hardware, operating system, or any component must be examined in the context of the total system. For example: It may not be possible to upgrade to a new version of Java because the database or CORBA solution does not yet support it.

\section{Conclusion}

PIMS is an information management system currently supporting the POIC's operational needs as the overall manager and integrator of ISS science payloads and experiments. It has been operational since March 2001. Even after PIMS became operational, the iterative development process has continued. Iterative improvements have significantly reduced system startup and recurring operational costs. Additionally, the system has adapted to better meet user needs with both new and altered capabilities. User satisfaction has continuously improved.

Sophisticated PIMS Java GUIs are capable of running over the Internet on virtually any common platform from a Web browser. Java GUIs enabled the clients to have the desired native application look and feel. PIMS is secure, scalable, adaptable, and configurable to alternative platforms, infrastructure implementations, and environments. These features are partly made possible by its multi-tier distributed object architecture built with common standards-based, ubiquitous technologies.

The generalized capabilities for change request processing and document configuration management make the system adaptable as an information repository and change request approval system not only for the POIC, but also for alternative purposes. In summary, PIMS provides the POIC universal information management system of generalized capabilities that are widely accessible and highly adaptable. 

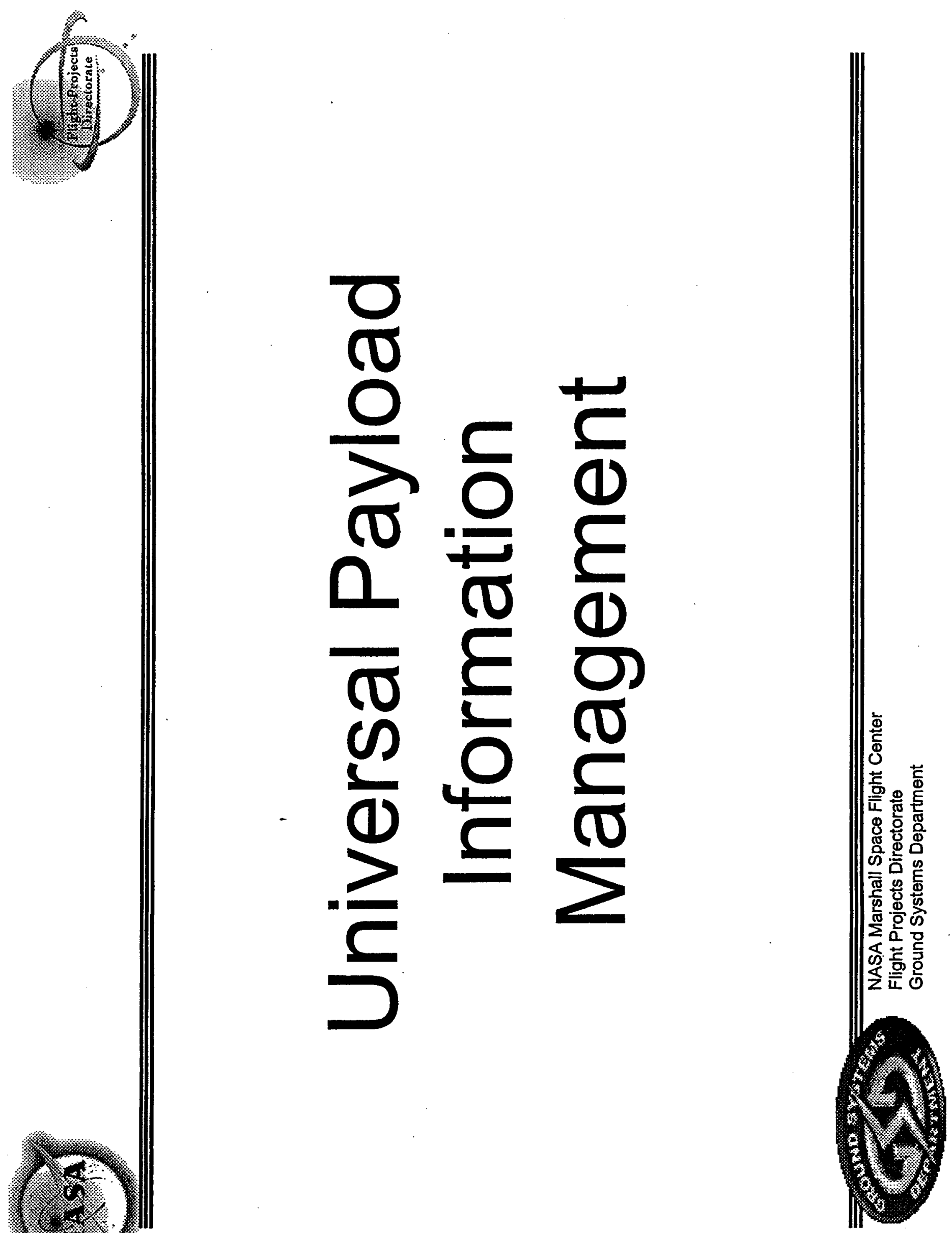


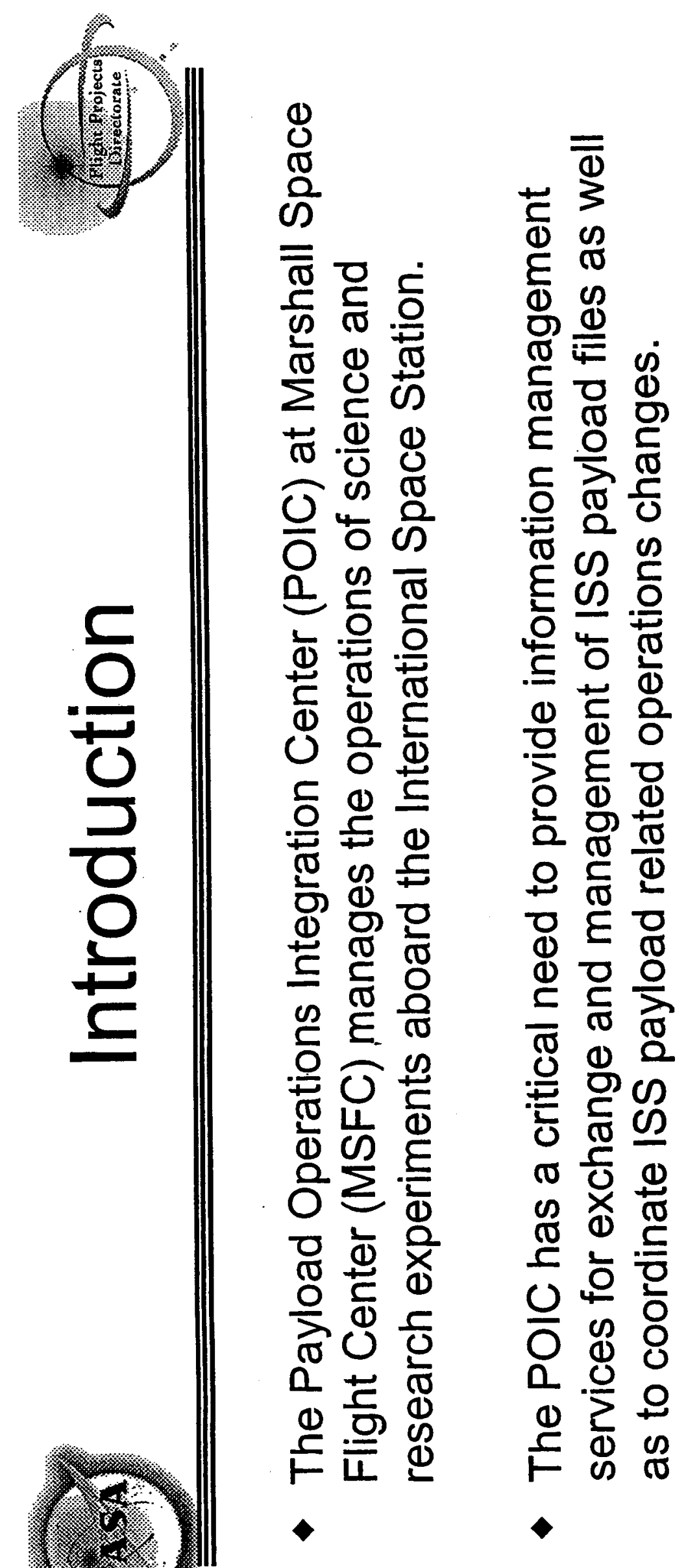

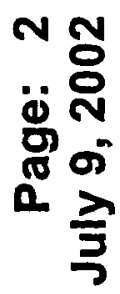

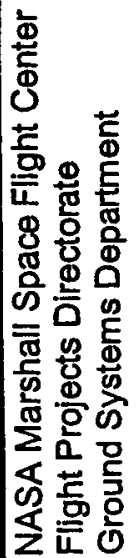



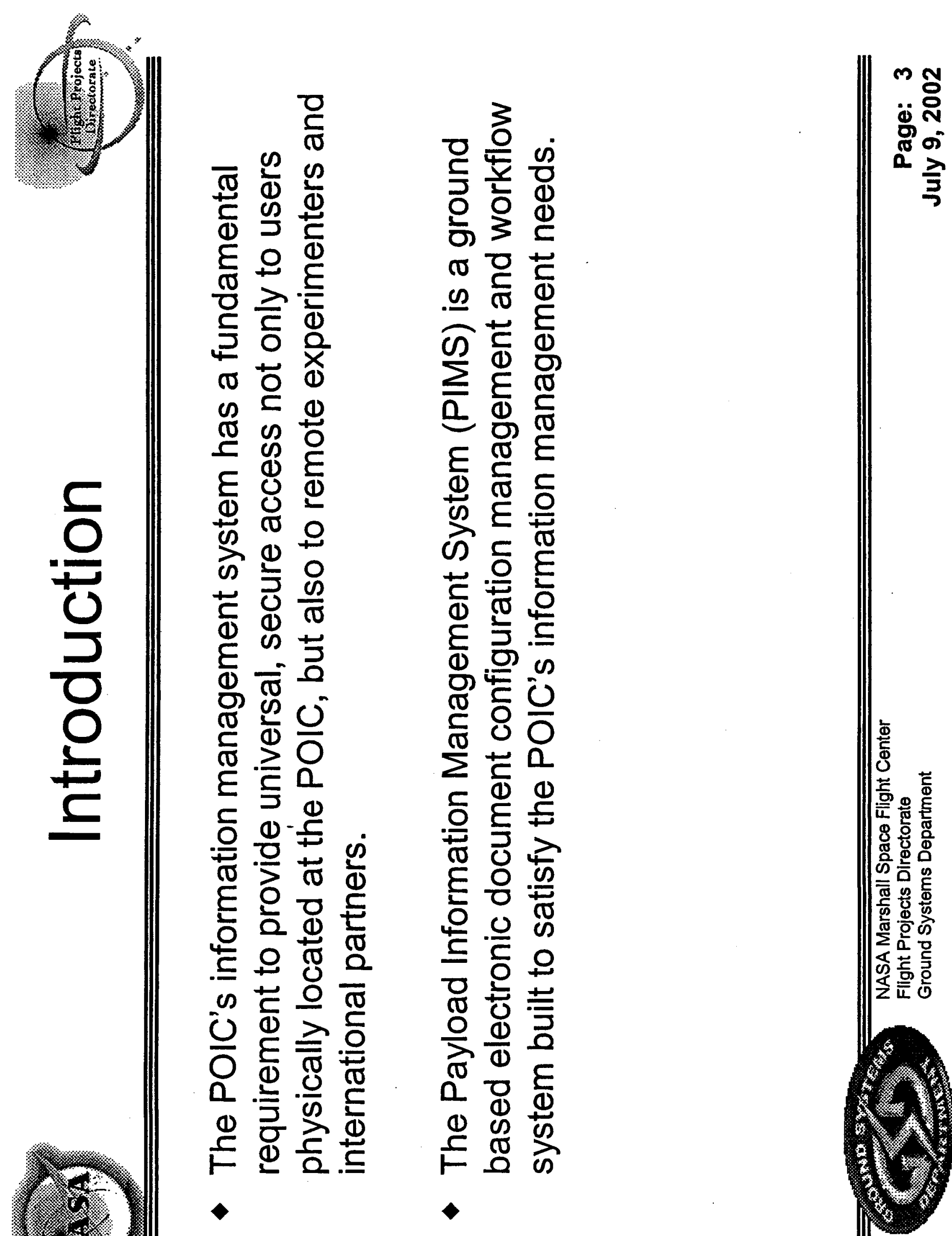


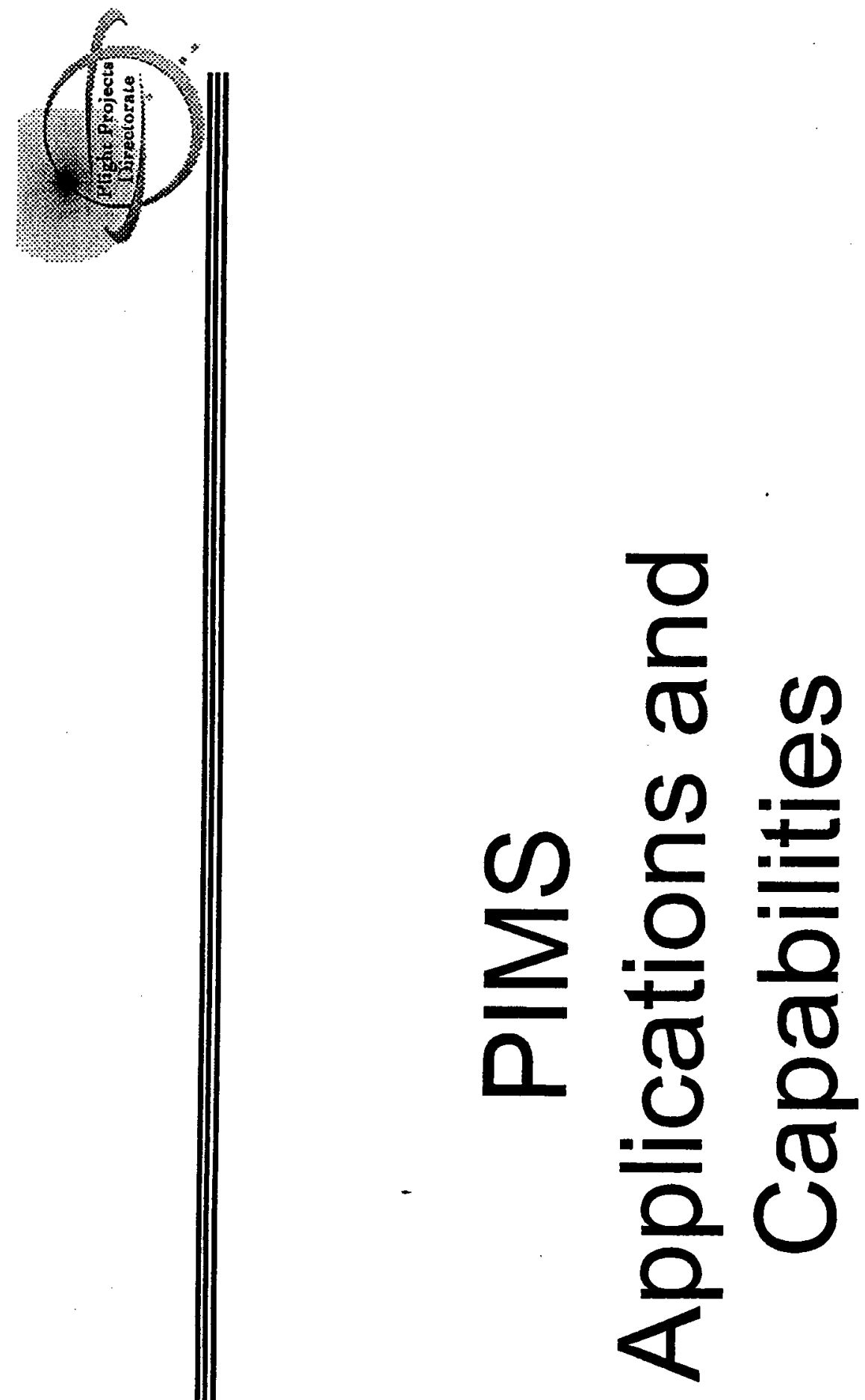




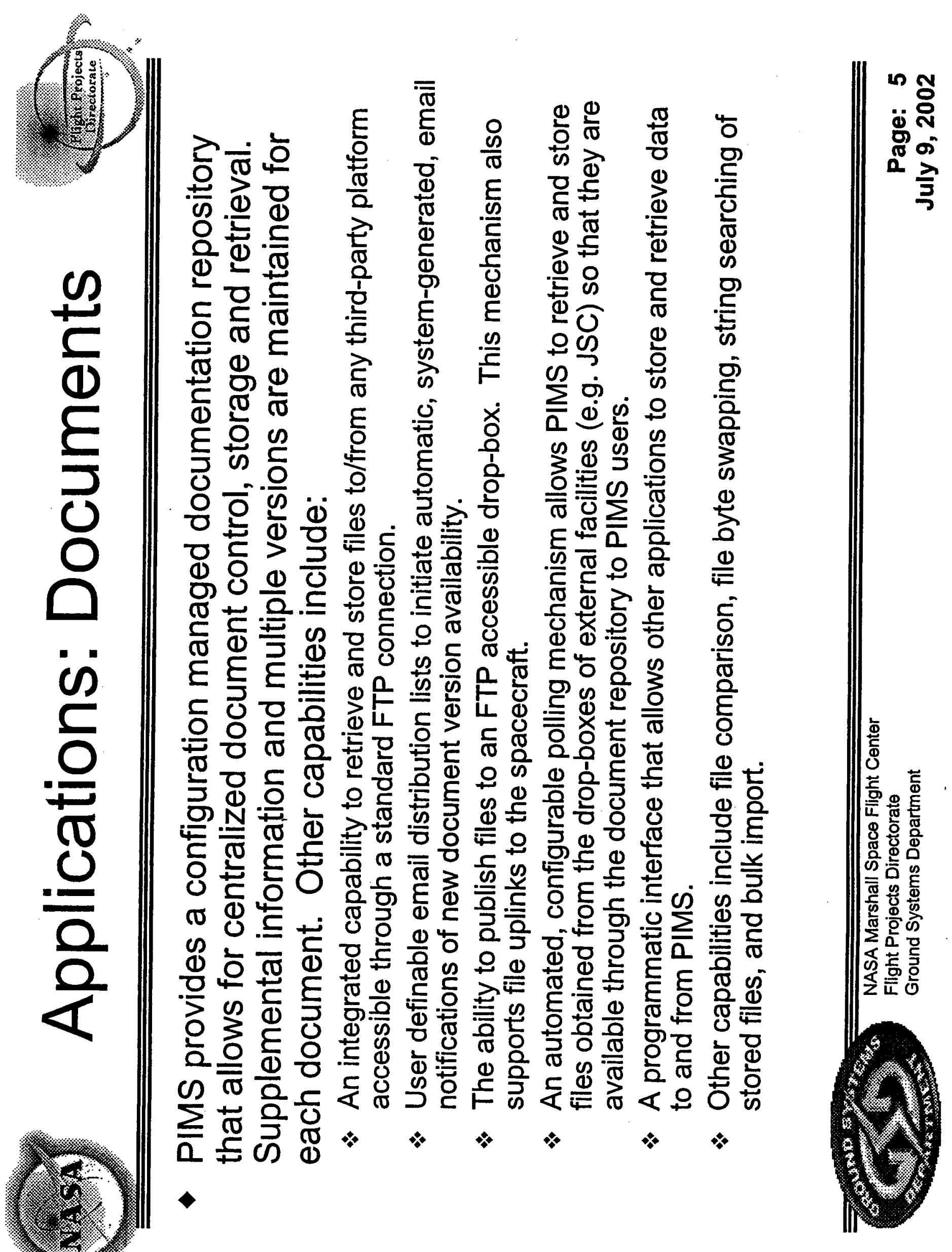




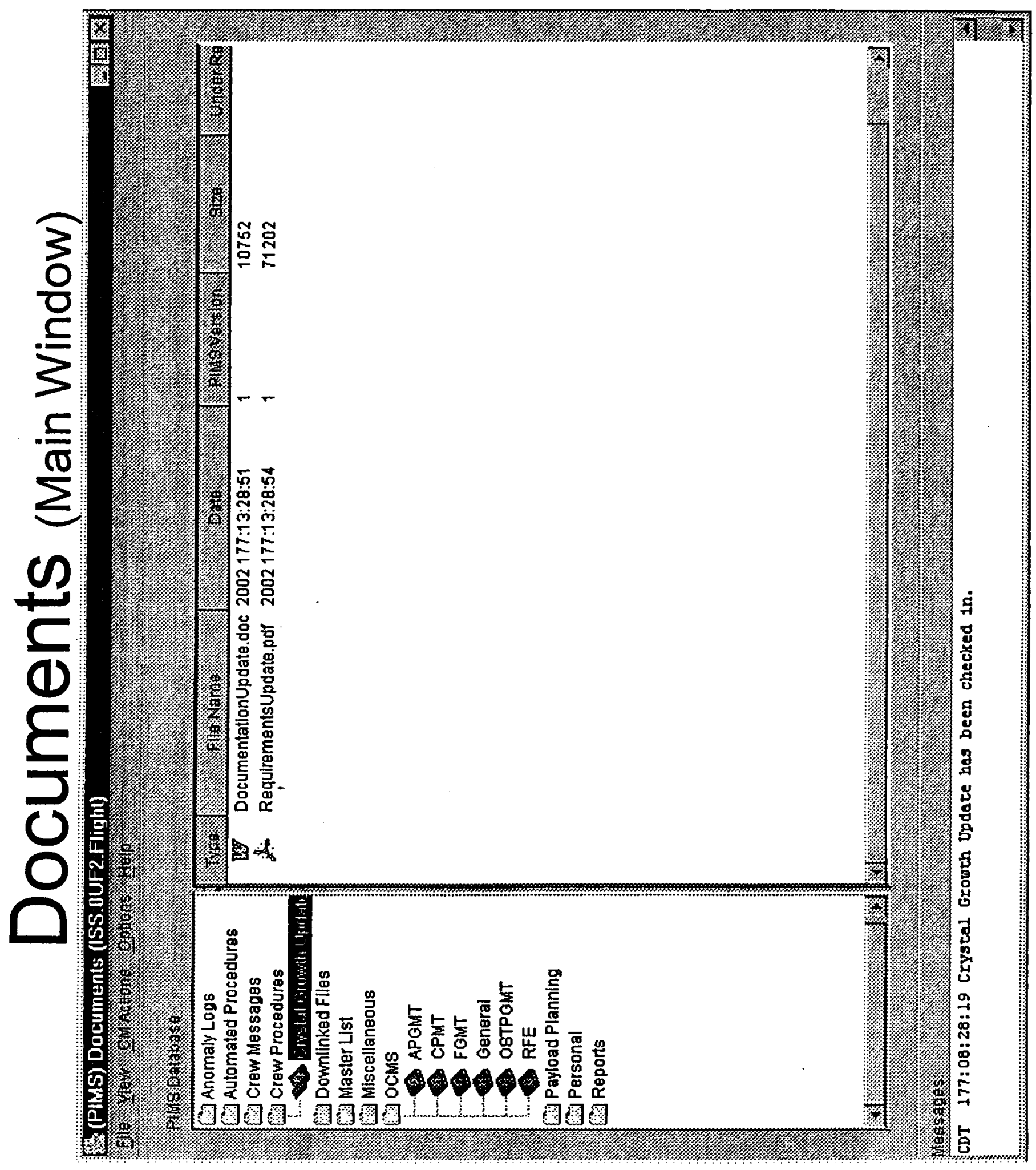




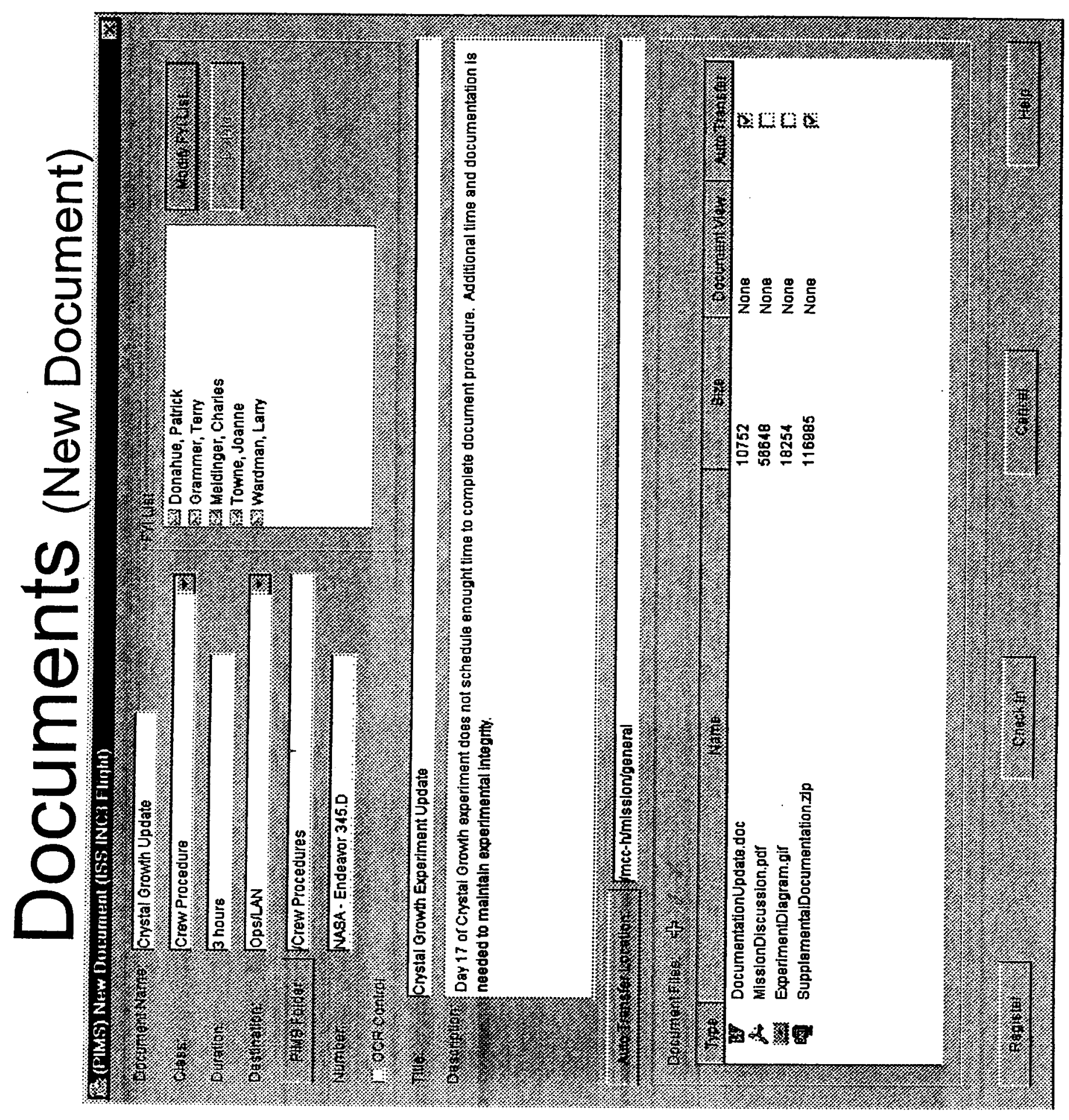




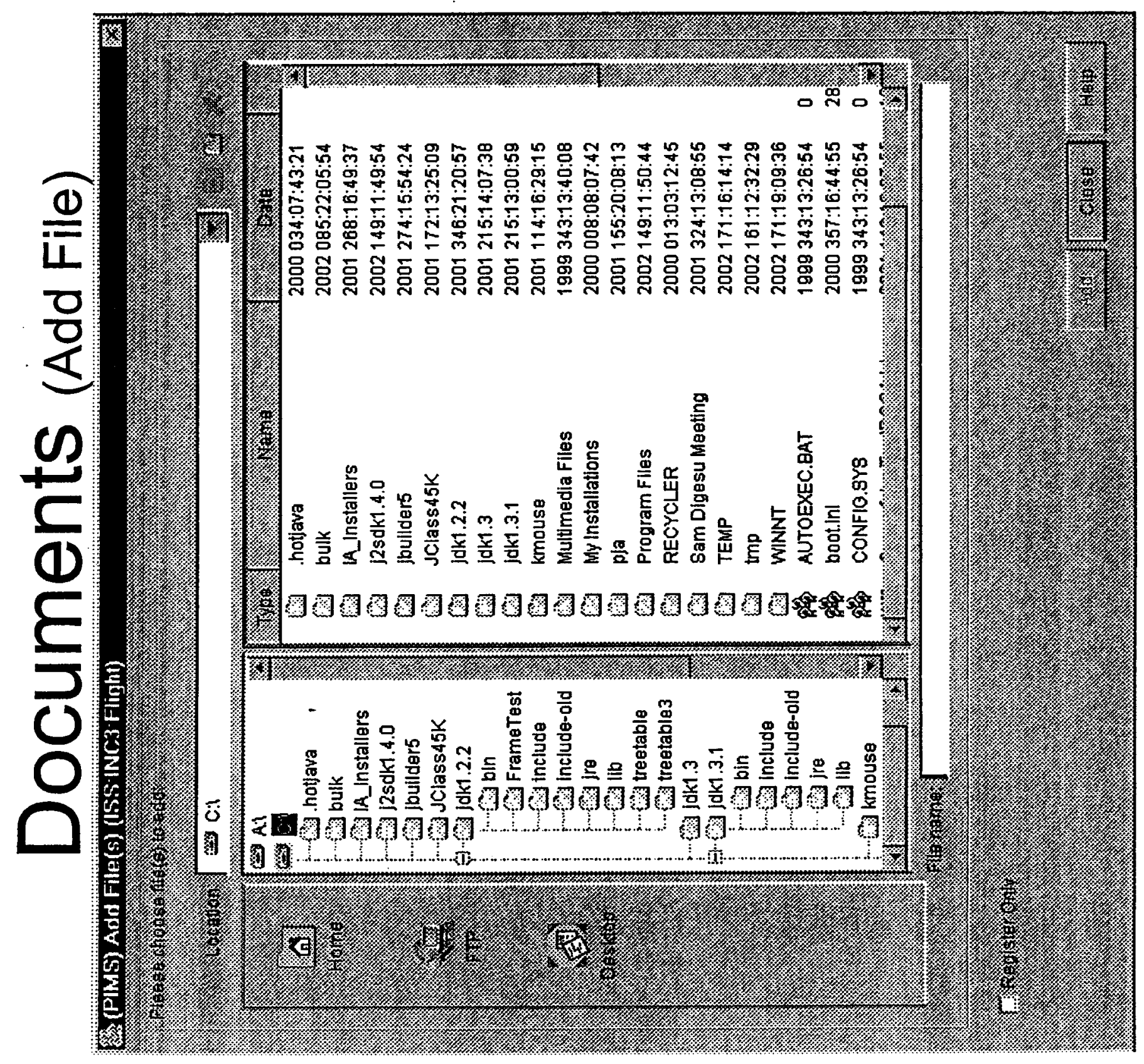




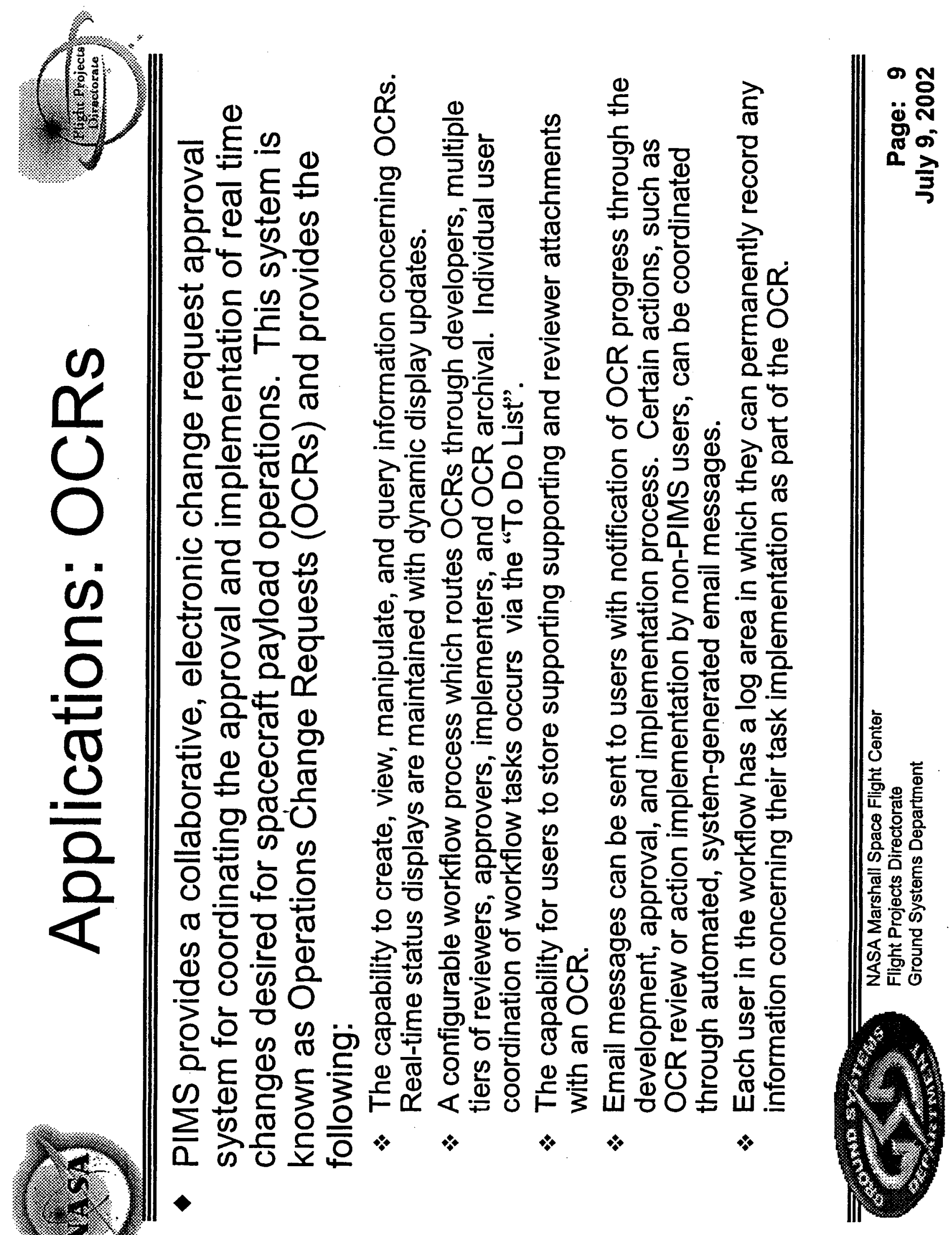




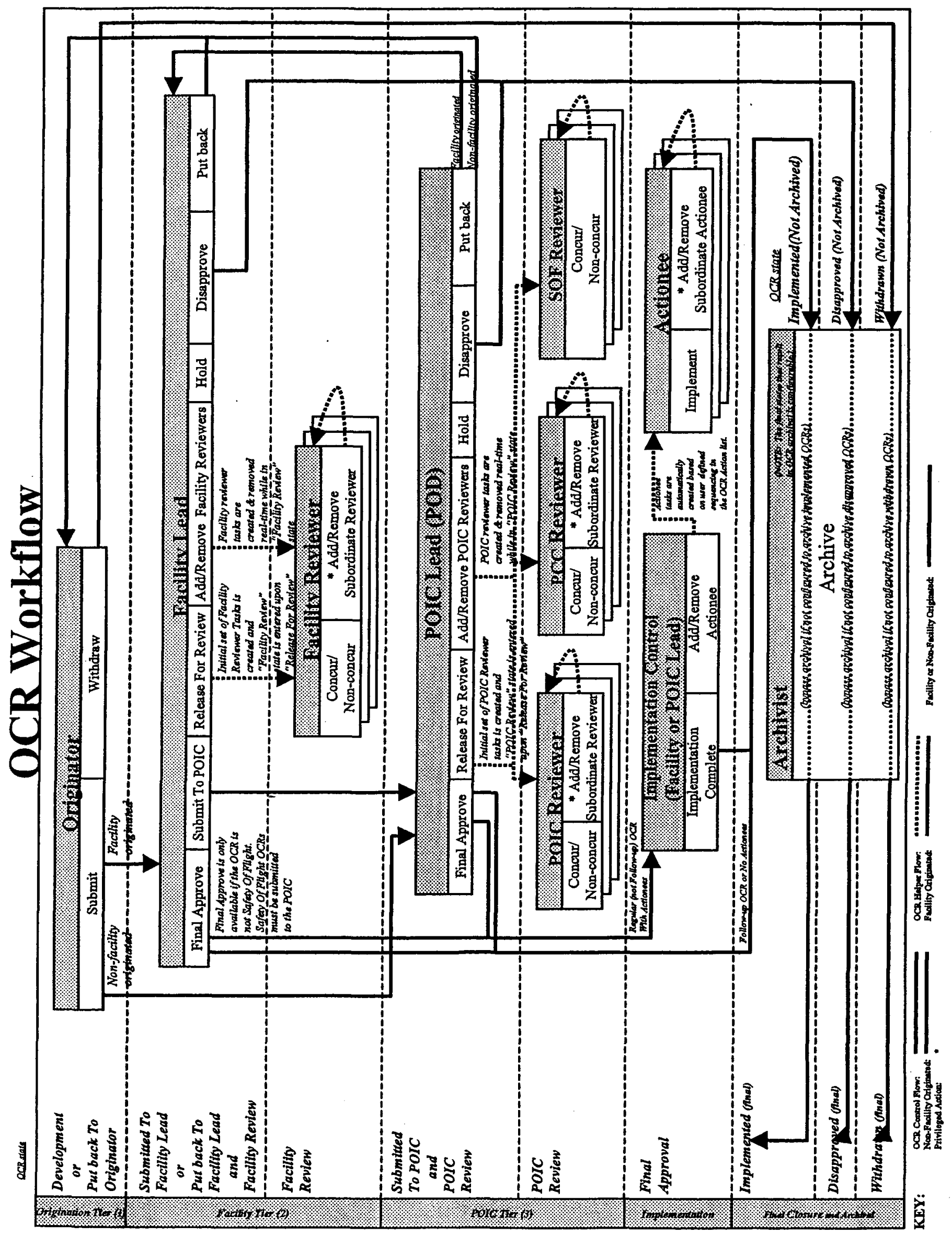



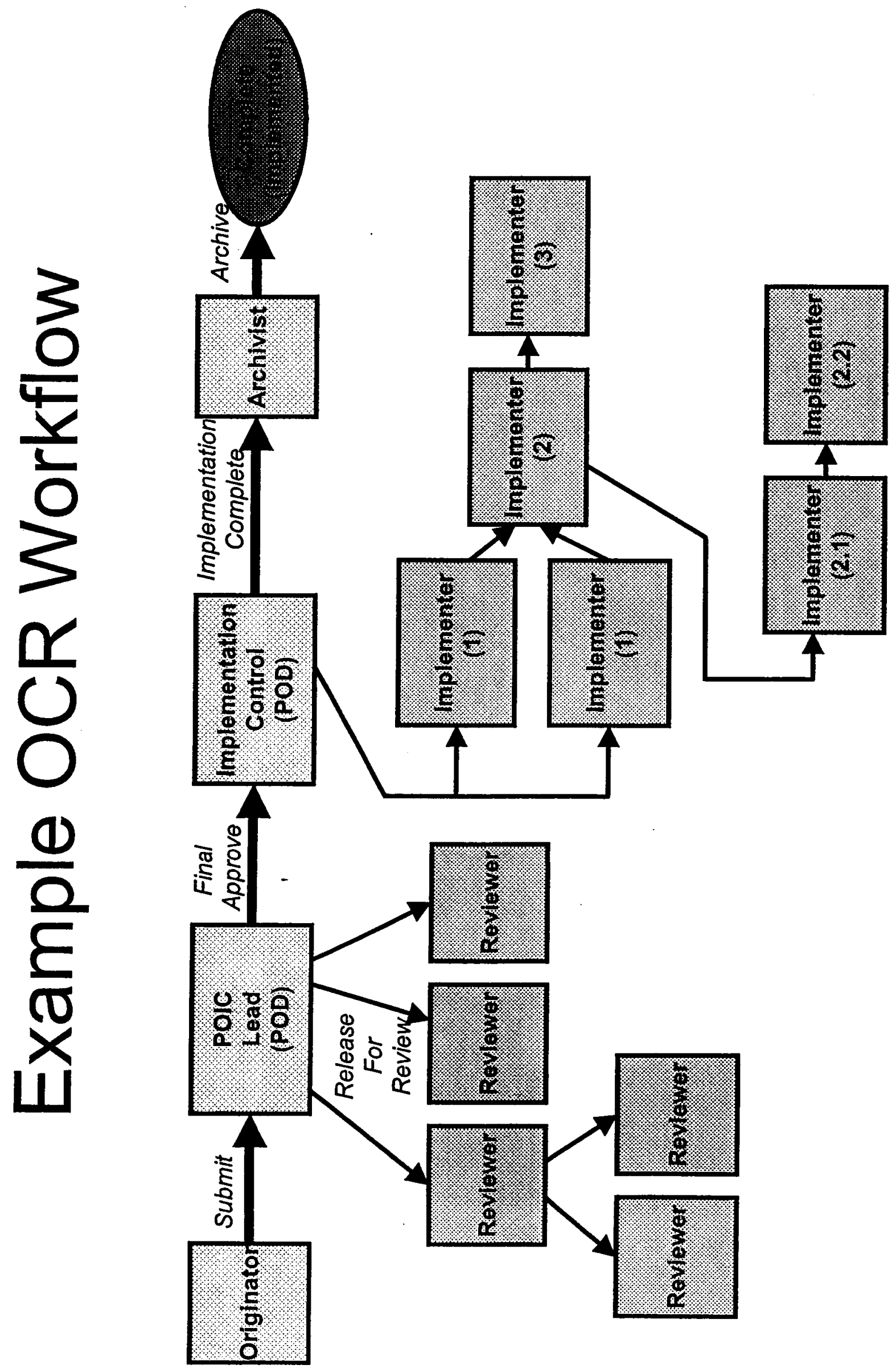


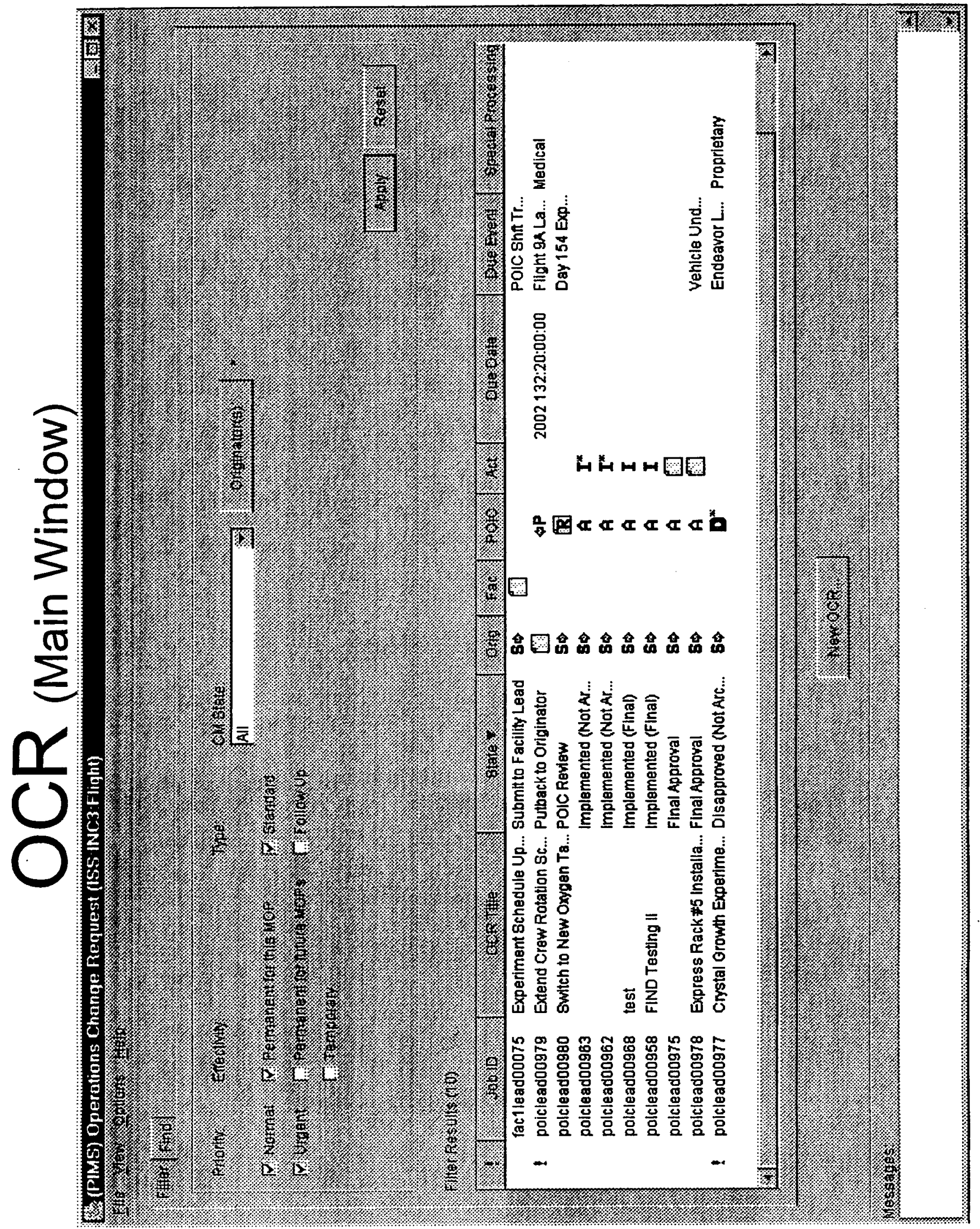




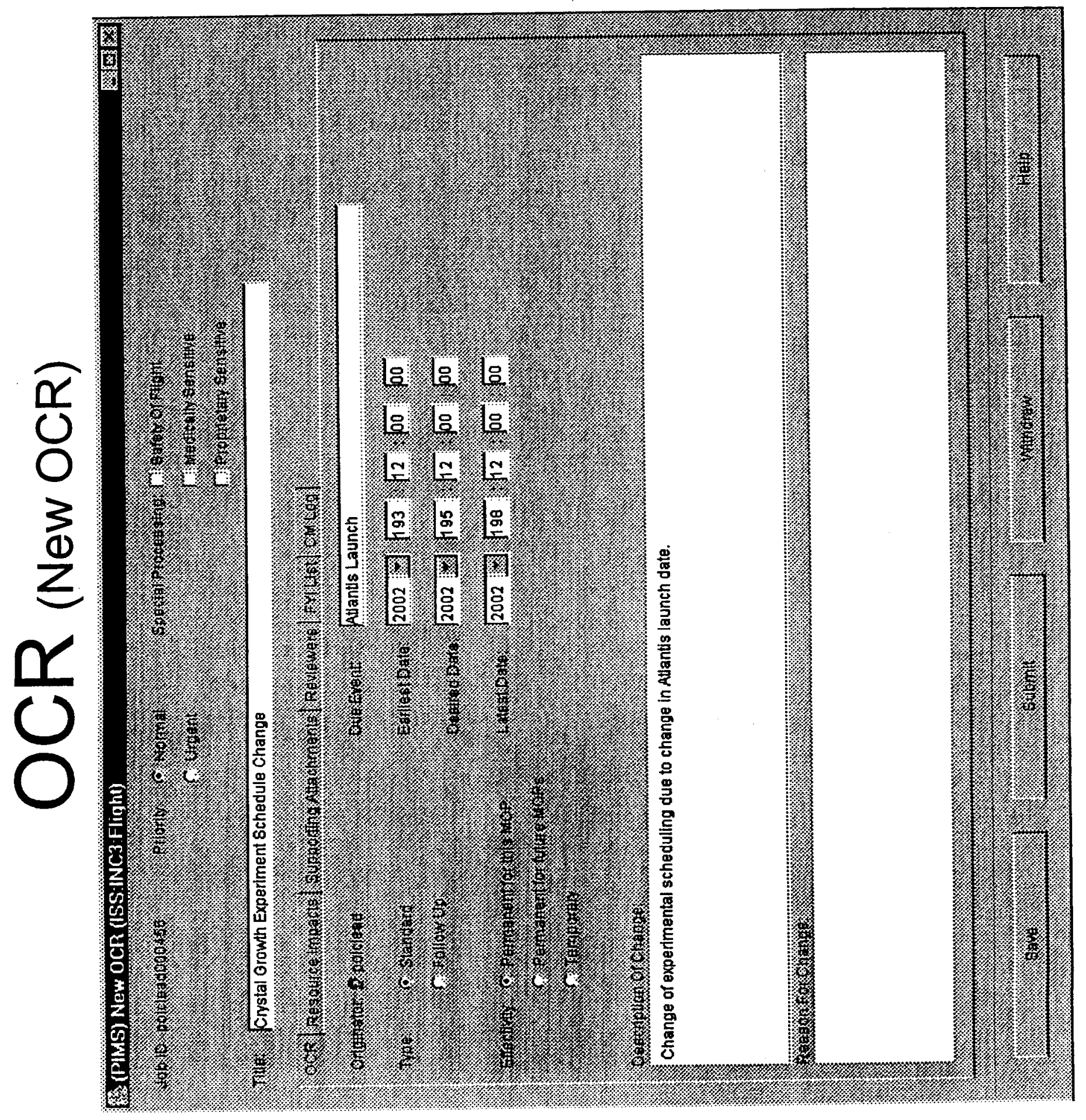




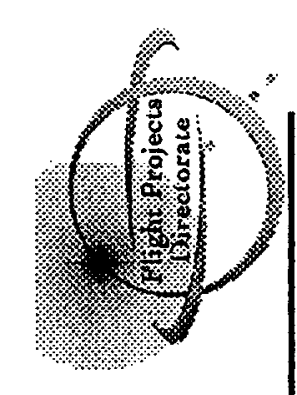

है

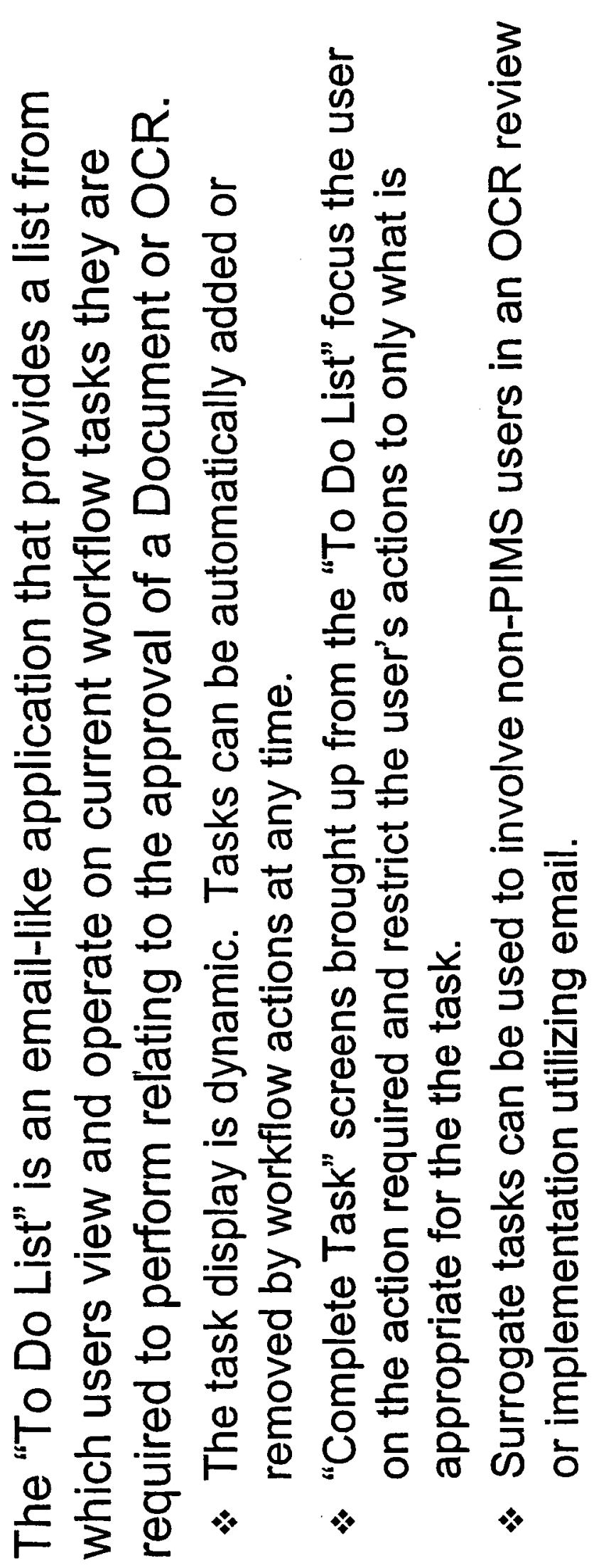

U 1 능

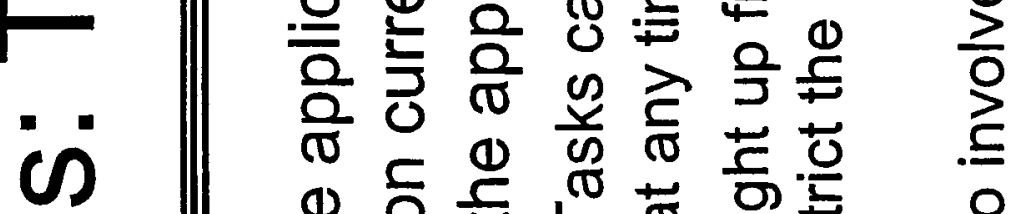

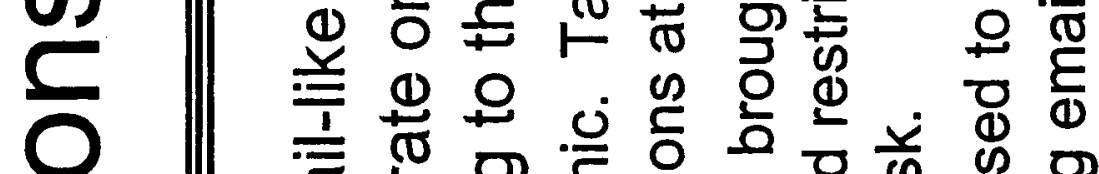

б

छ

QD

U

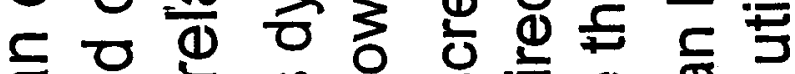

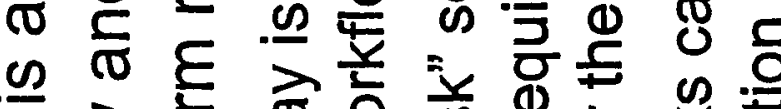

일

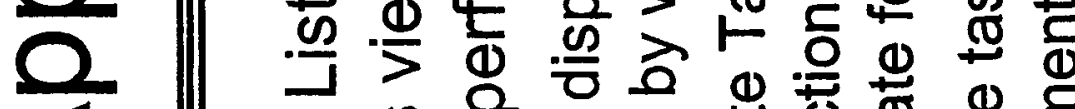

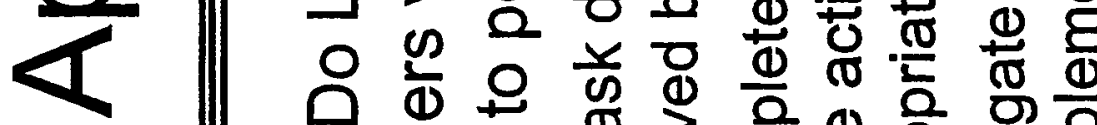

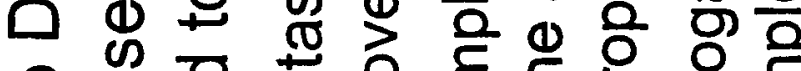

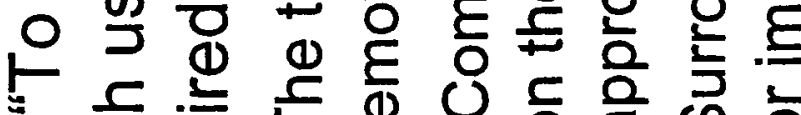

过

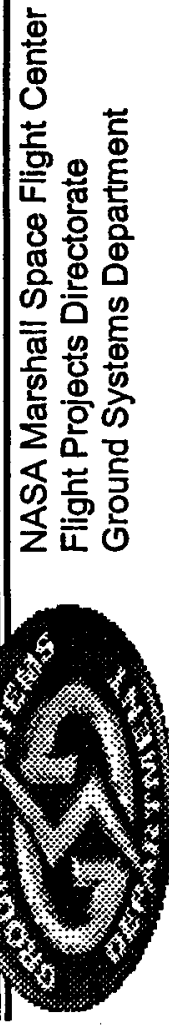




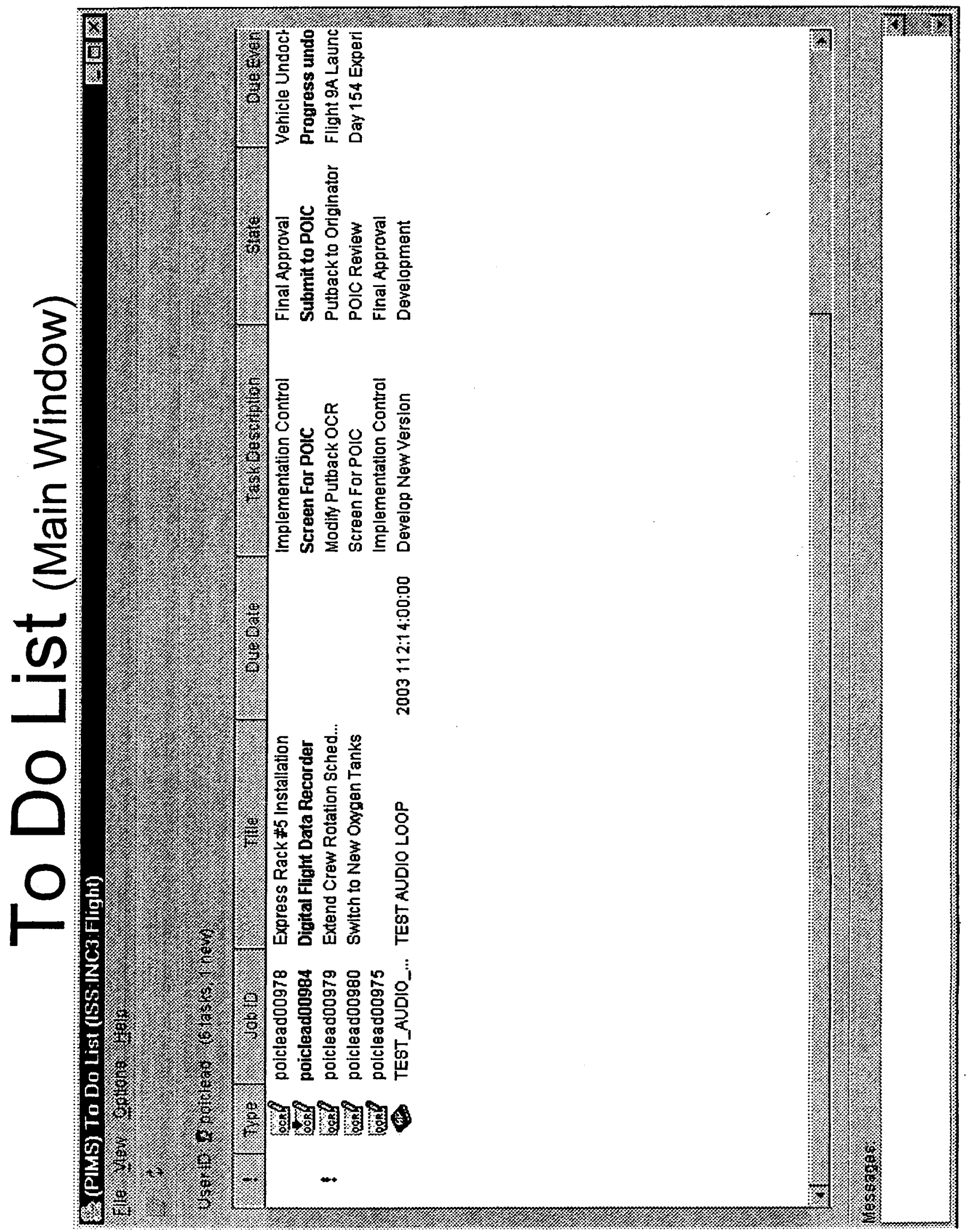




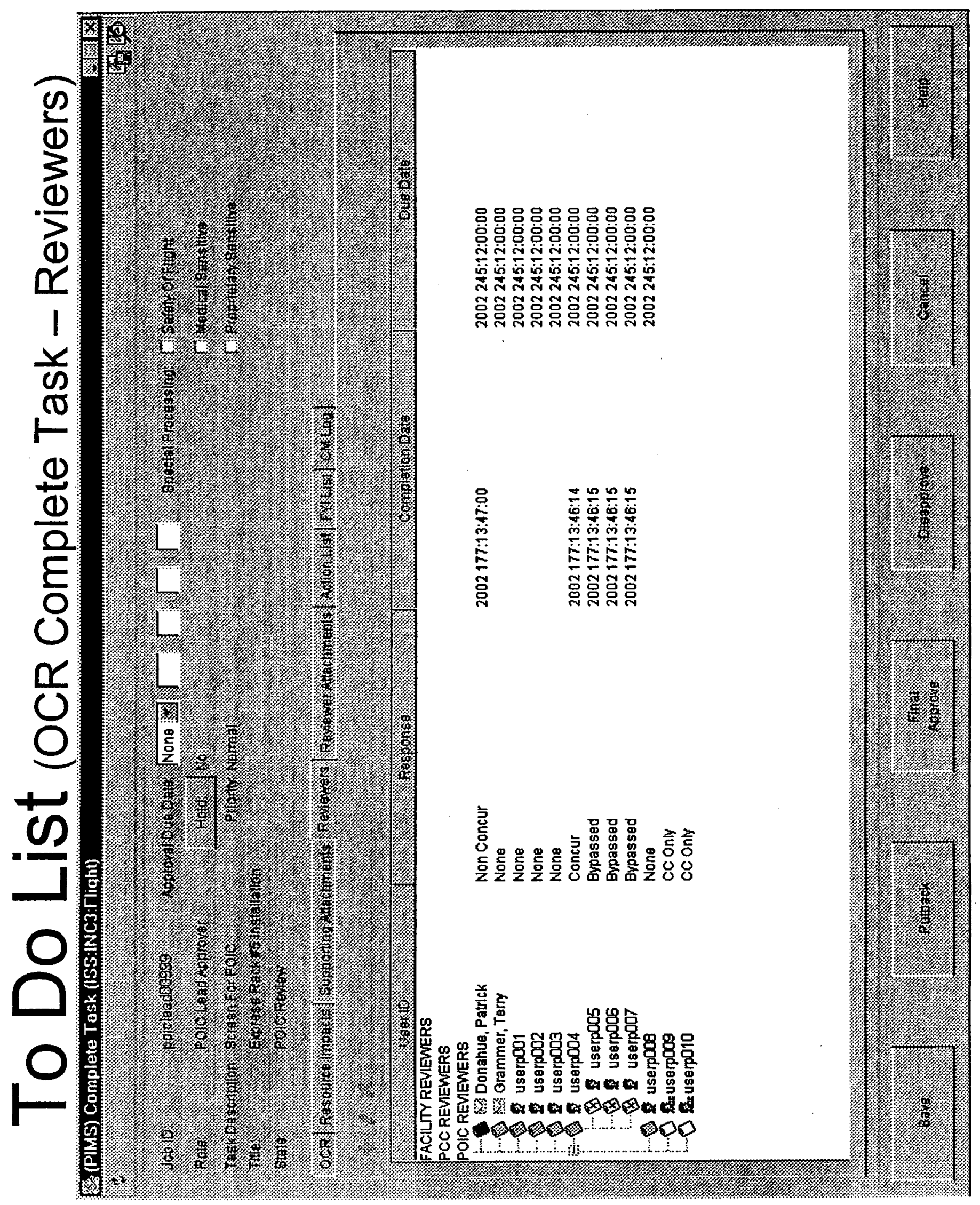




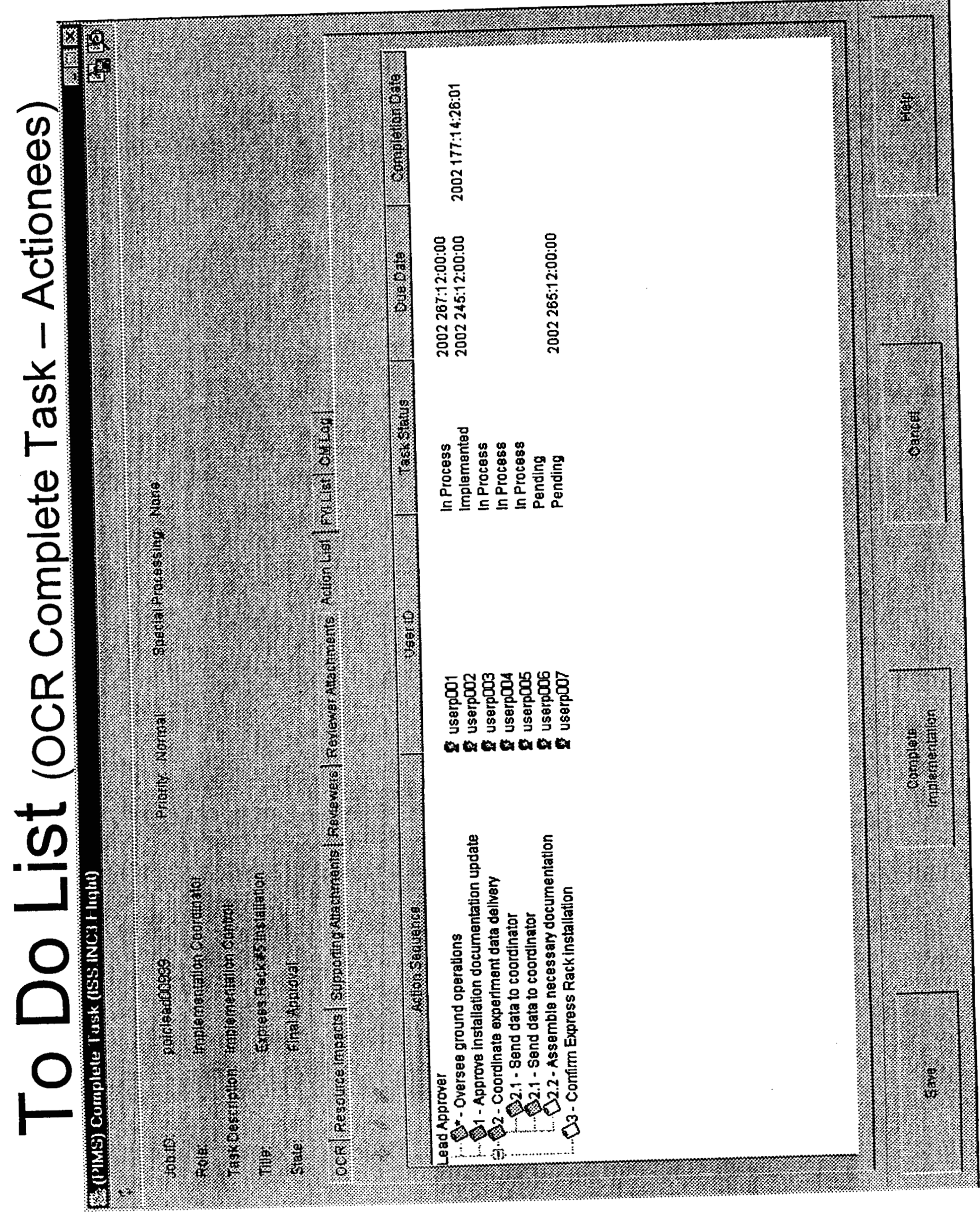




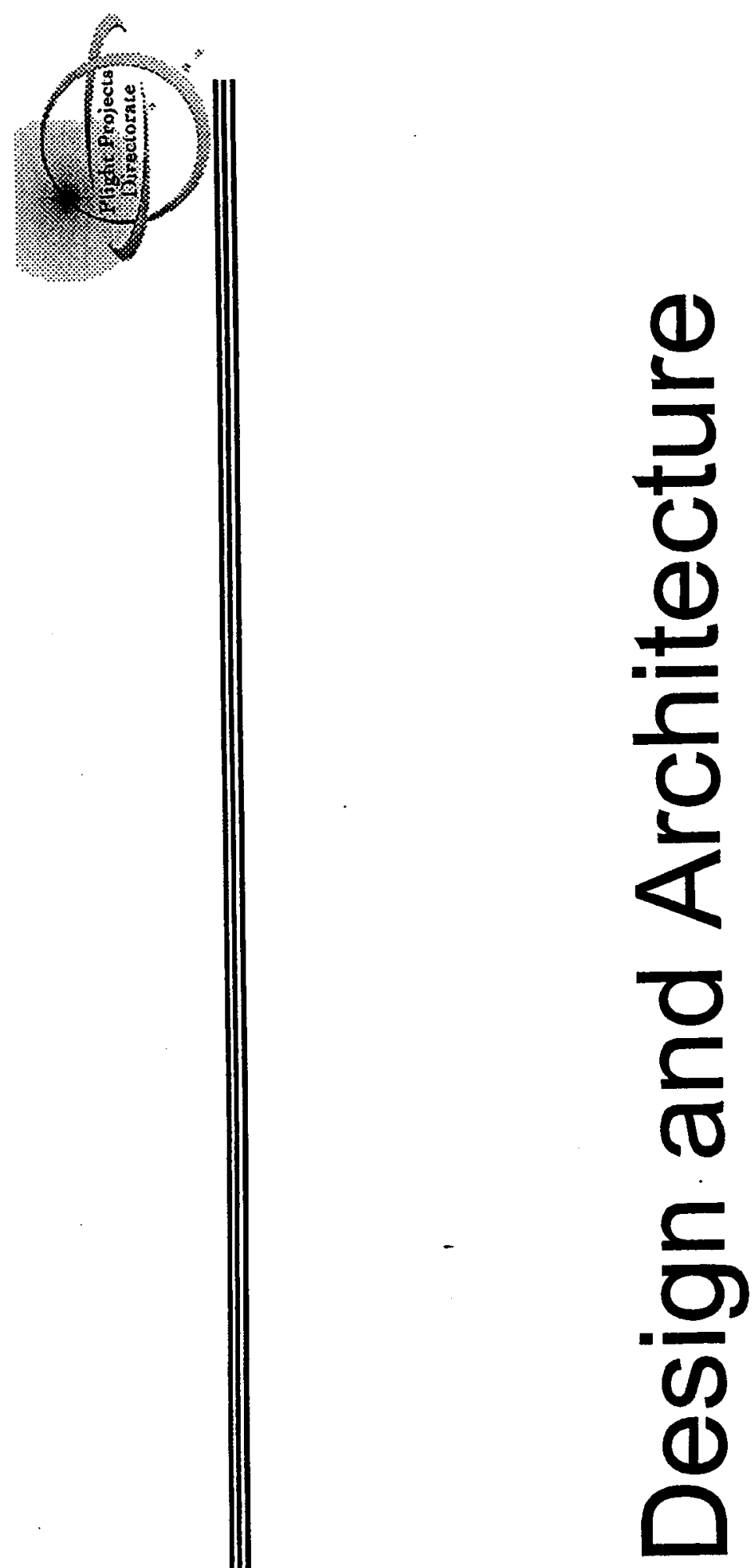

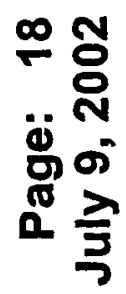

矛

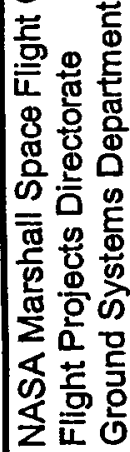




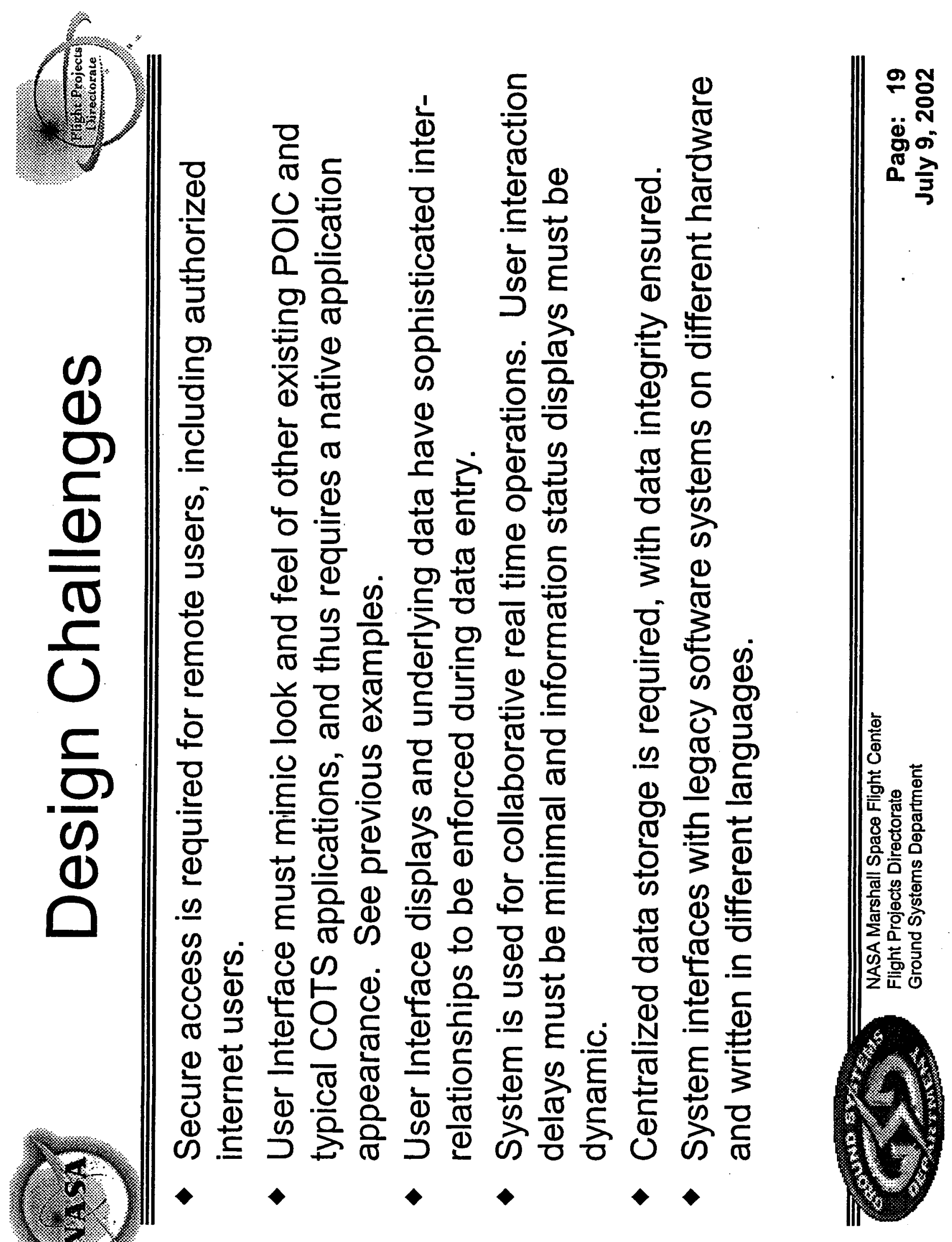




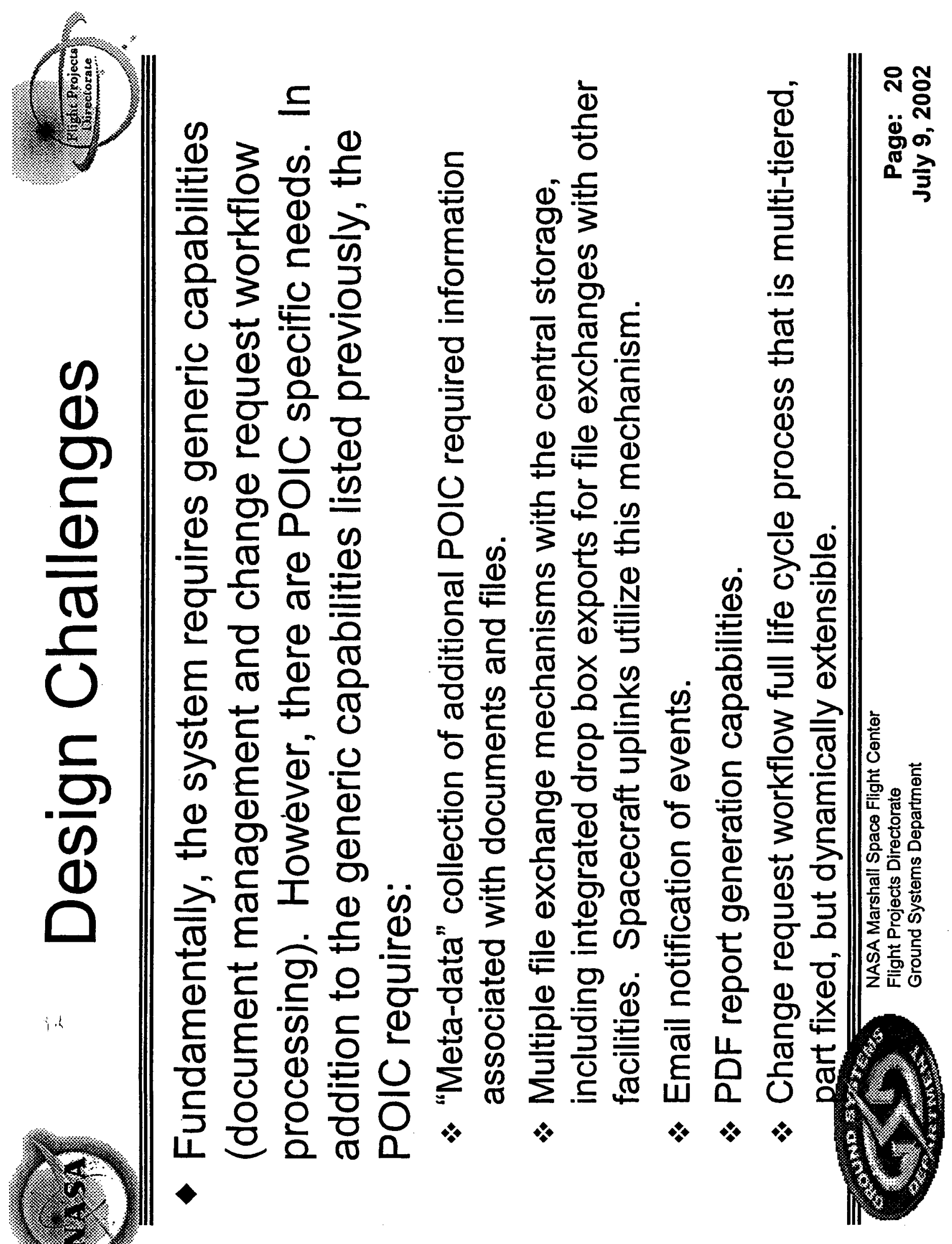




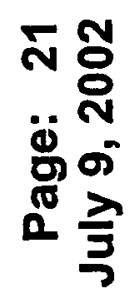

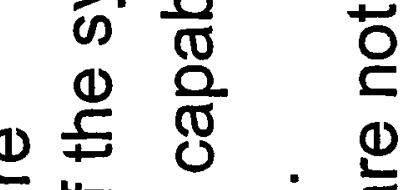

(1) $\frac{1}{0} \dot{0}$ 它

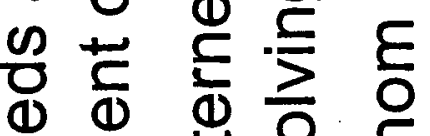

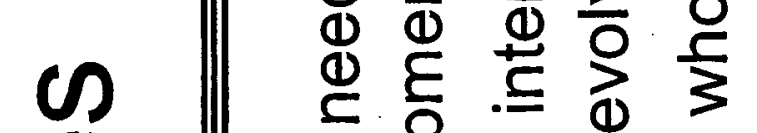

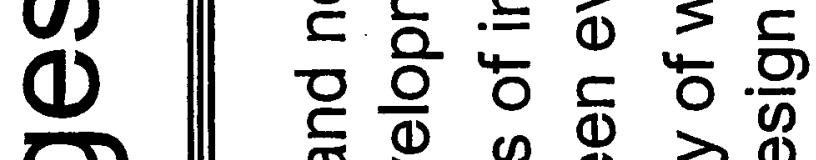

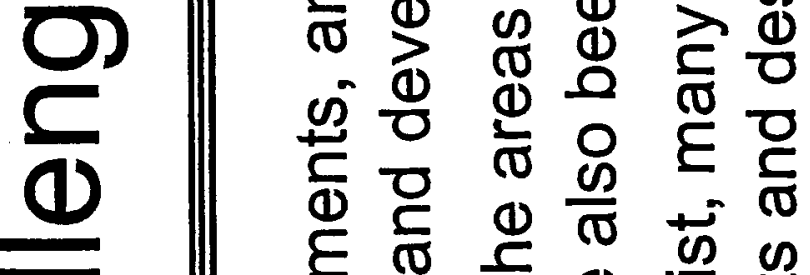

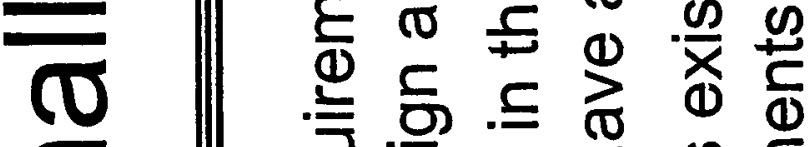

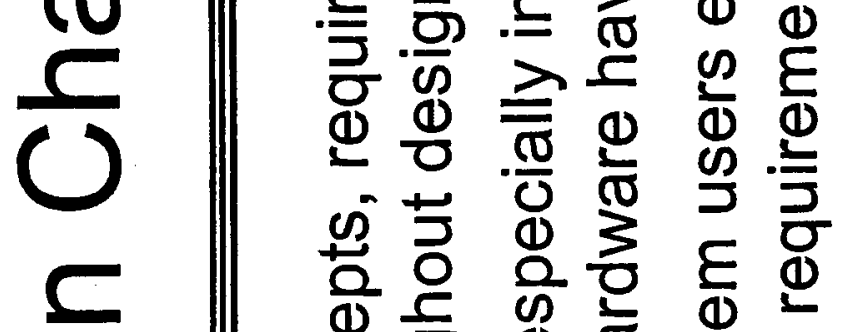

(D)

- 10 हु

0 至

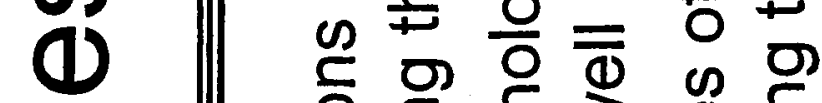

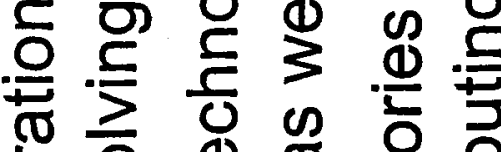

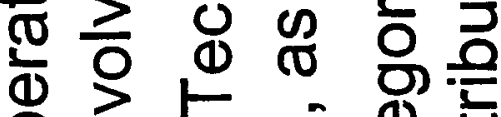

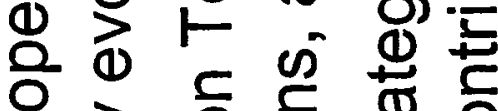

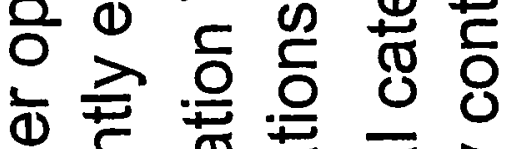

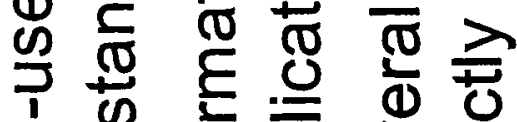

i $\frac{1}{0}$ 등

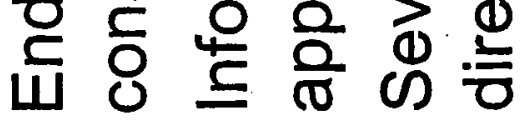




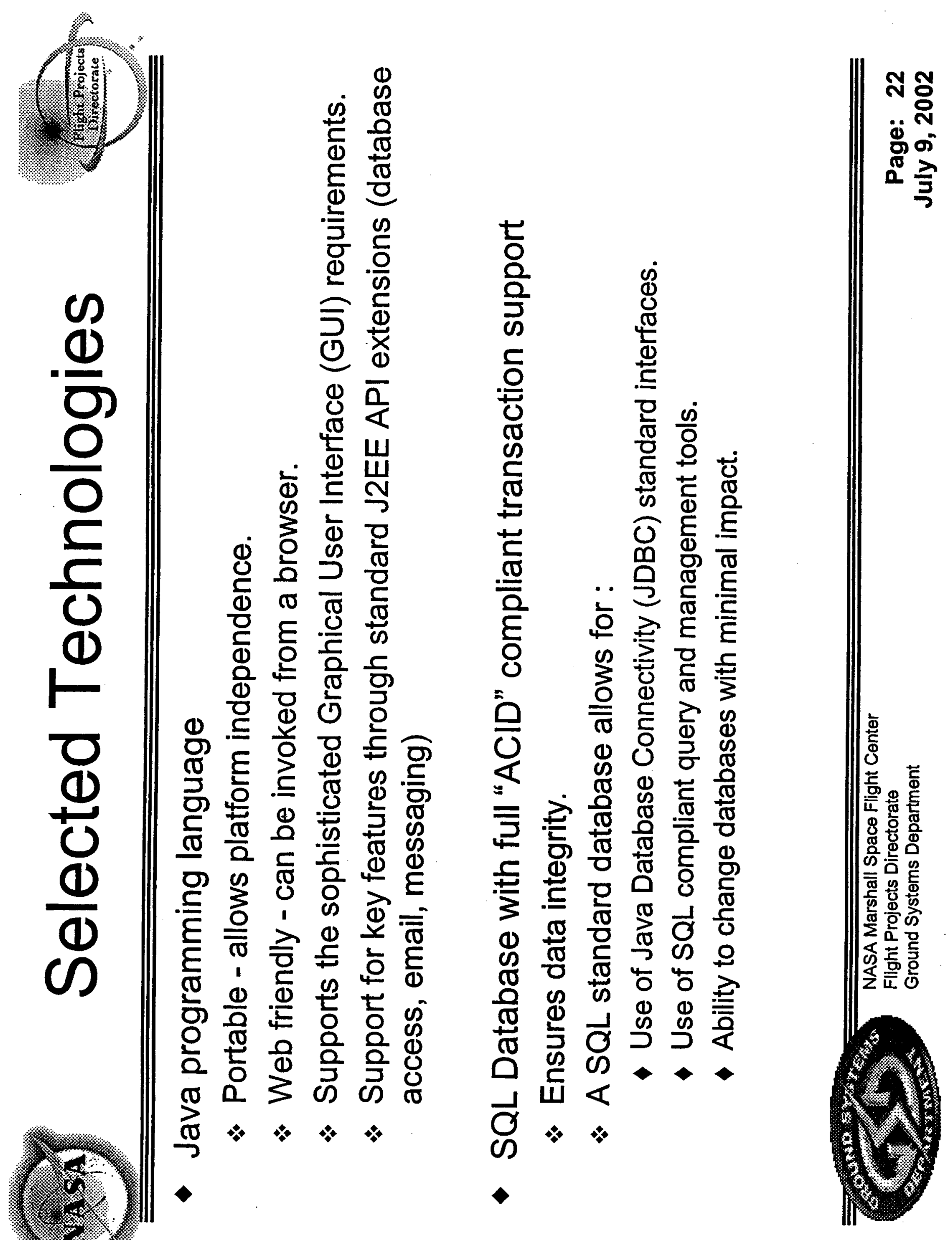



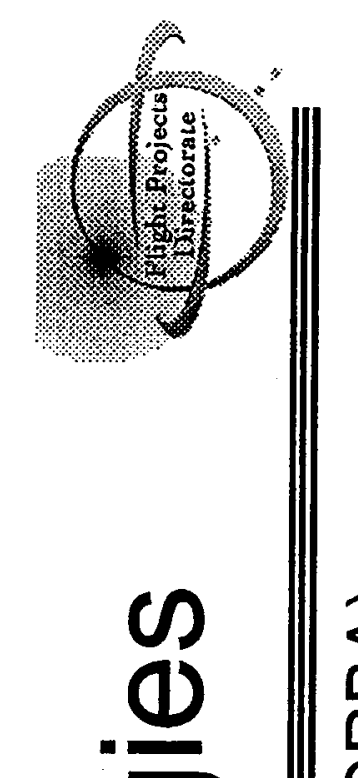

$$
\stackrel{5}{2}
$$

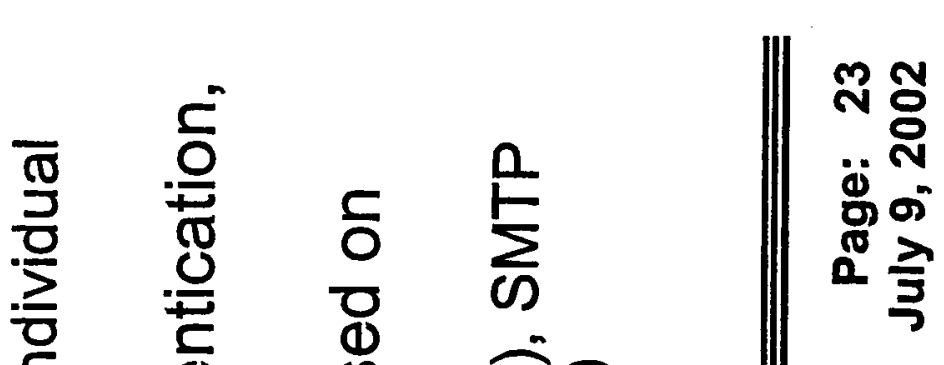

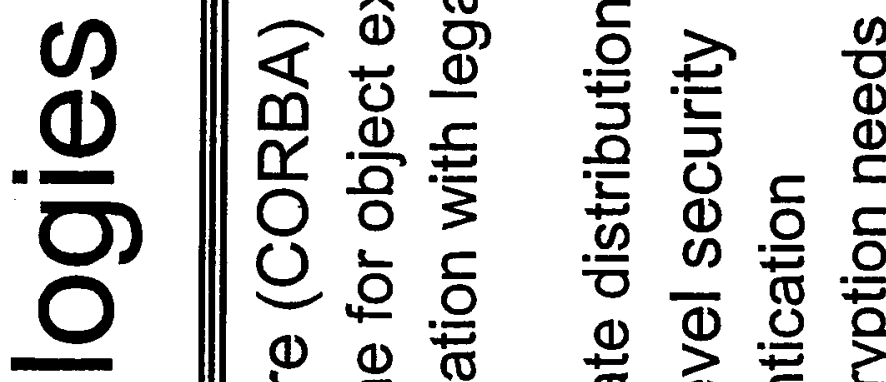

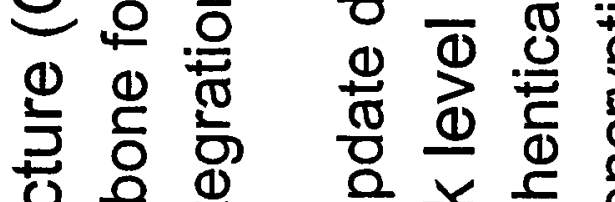

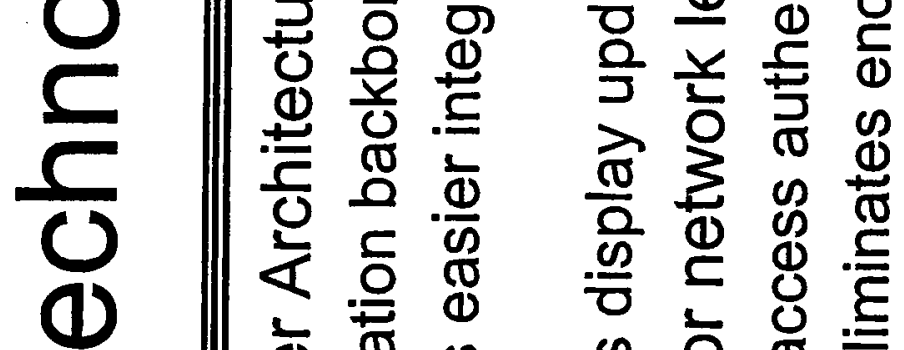

$\vdash$

(1) 임 壬 읗

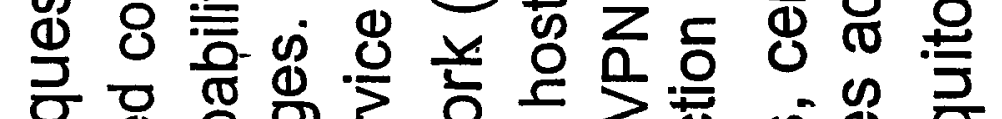

0

(1) ब 舟

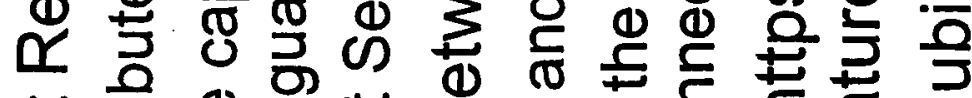

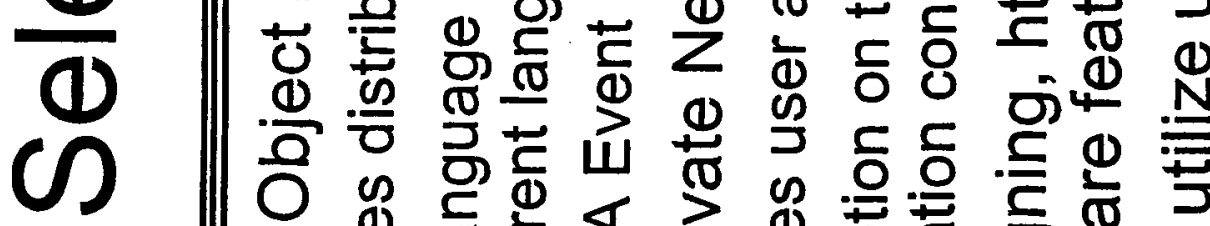
О

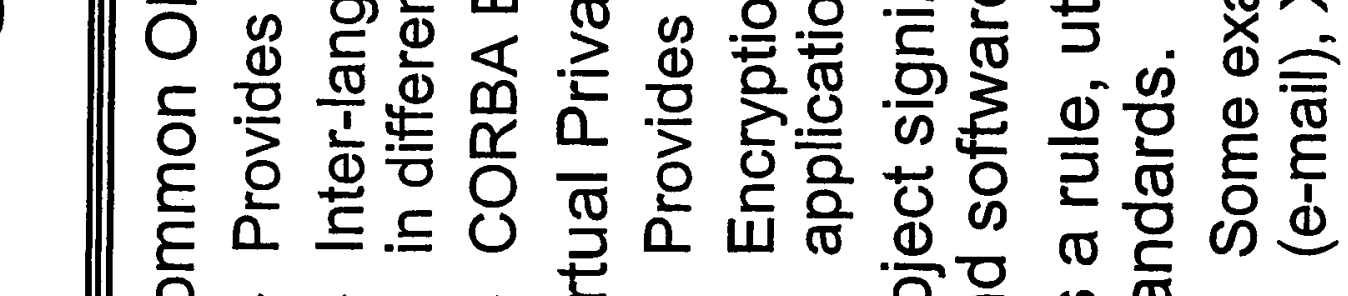

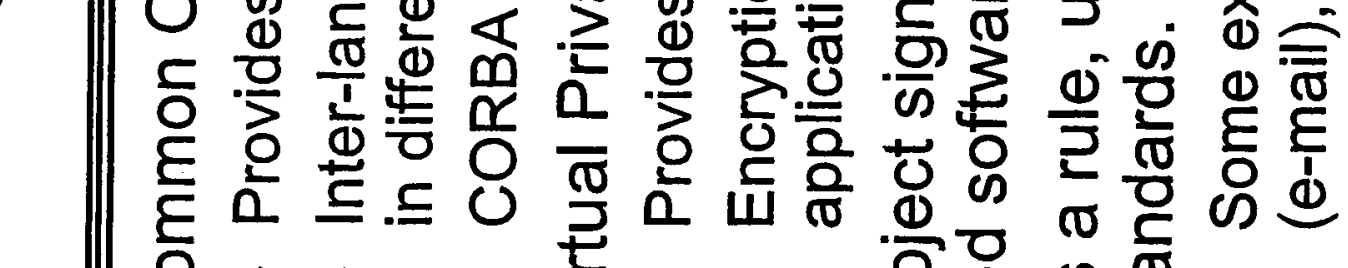

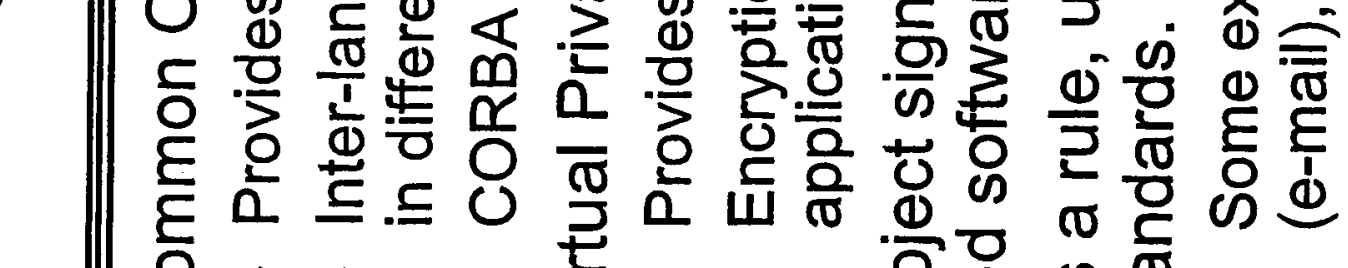
O * * * * O O 멍 용 急 랄 สำ 


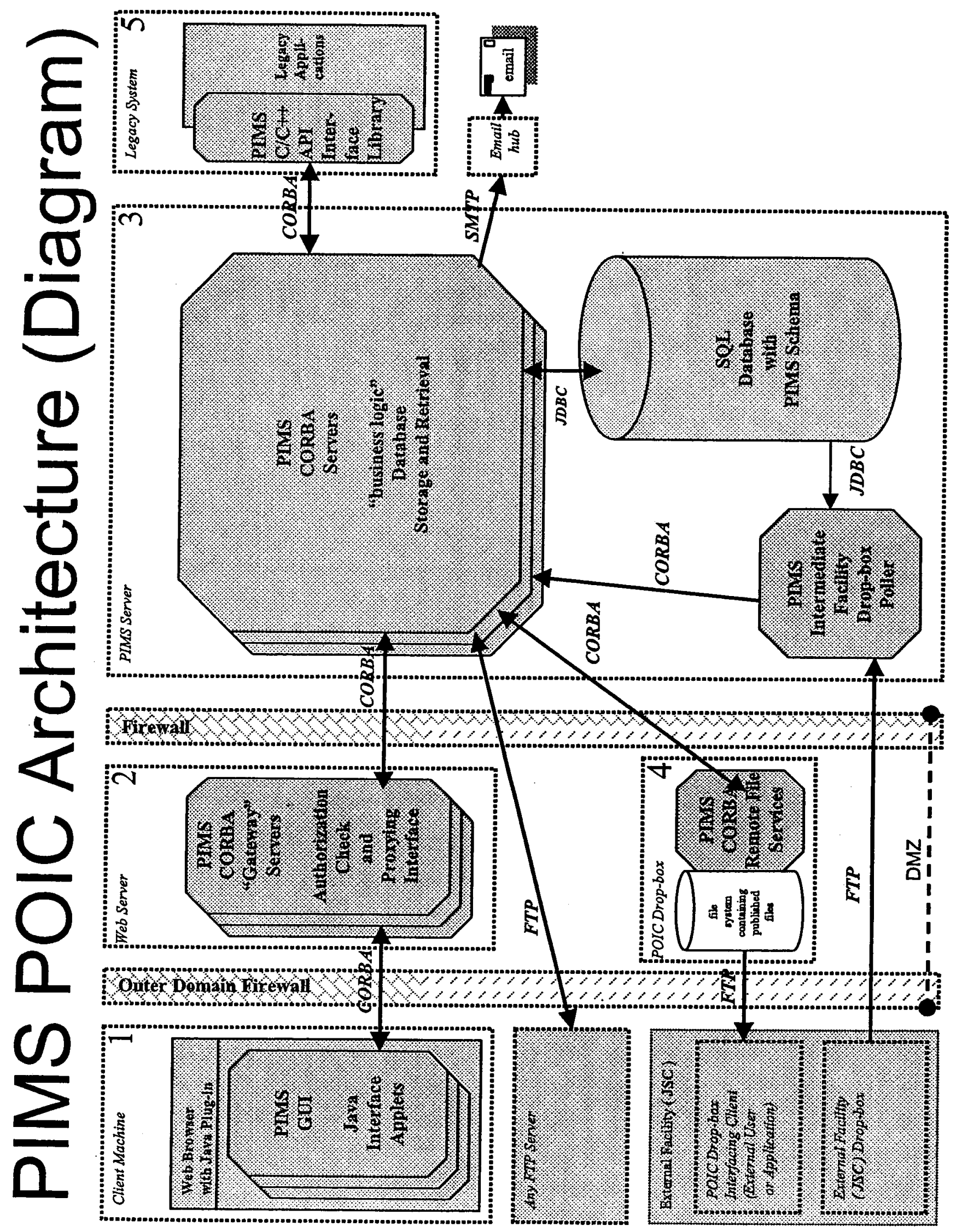




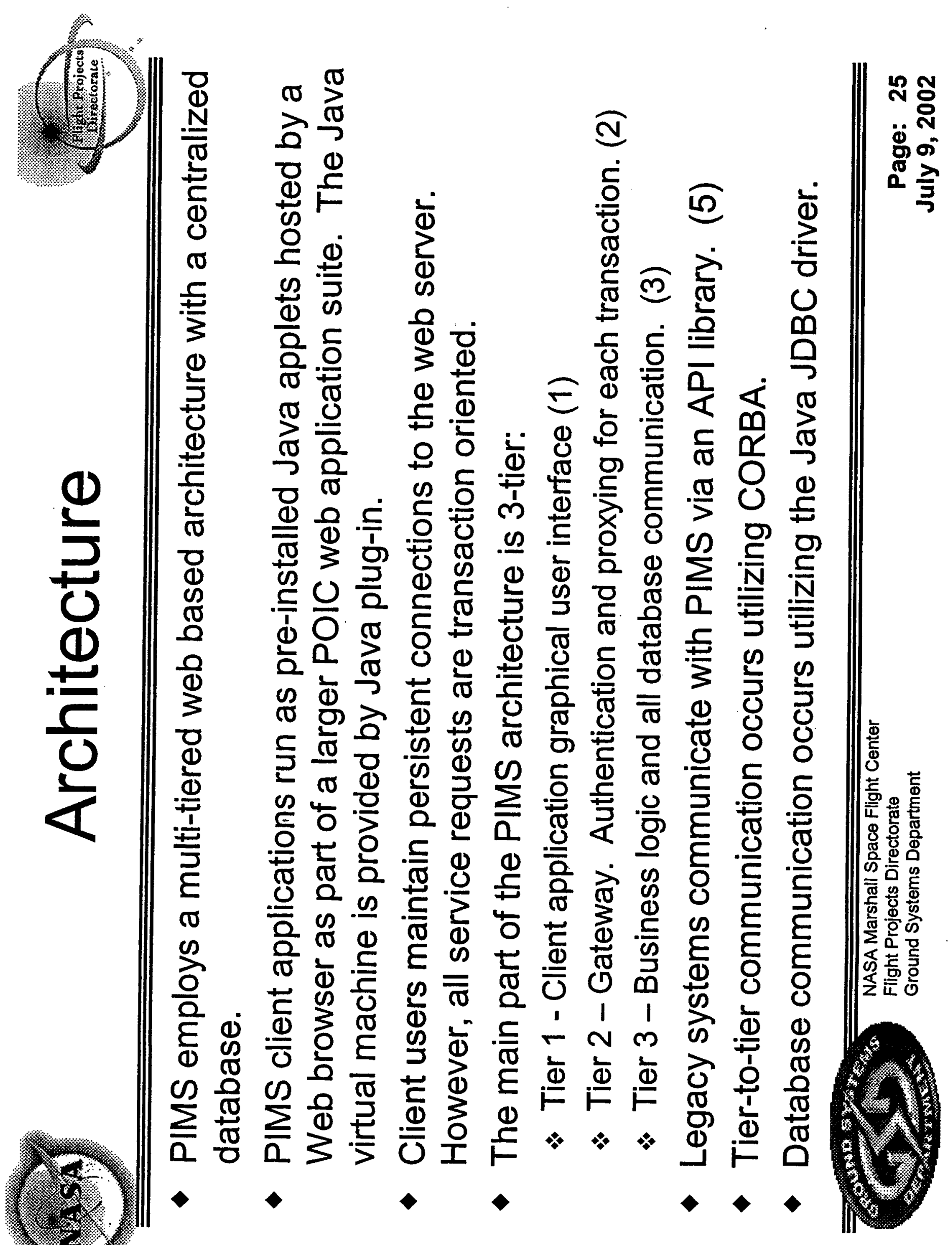




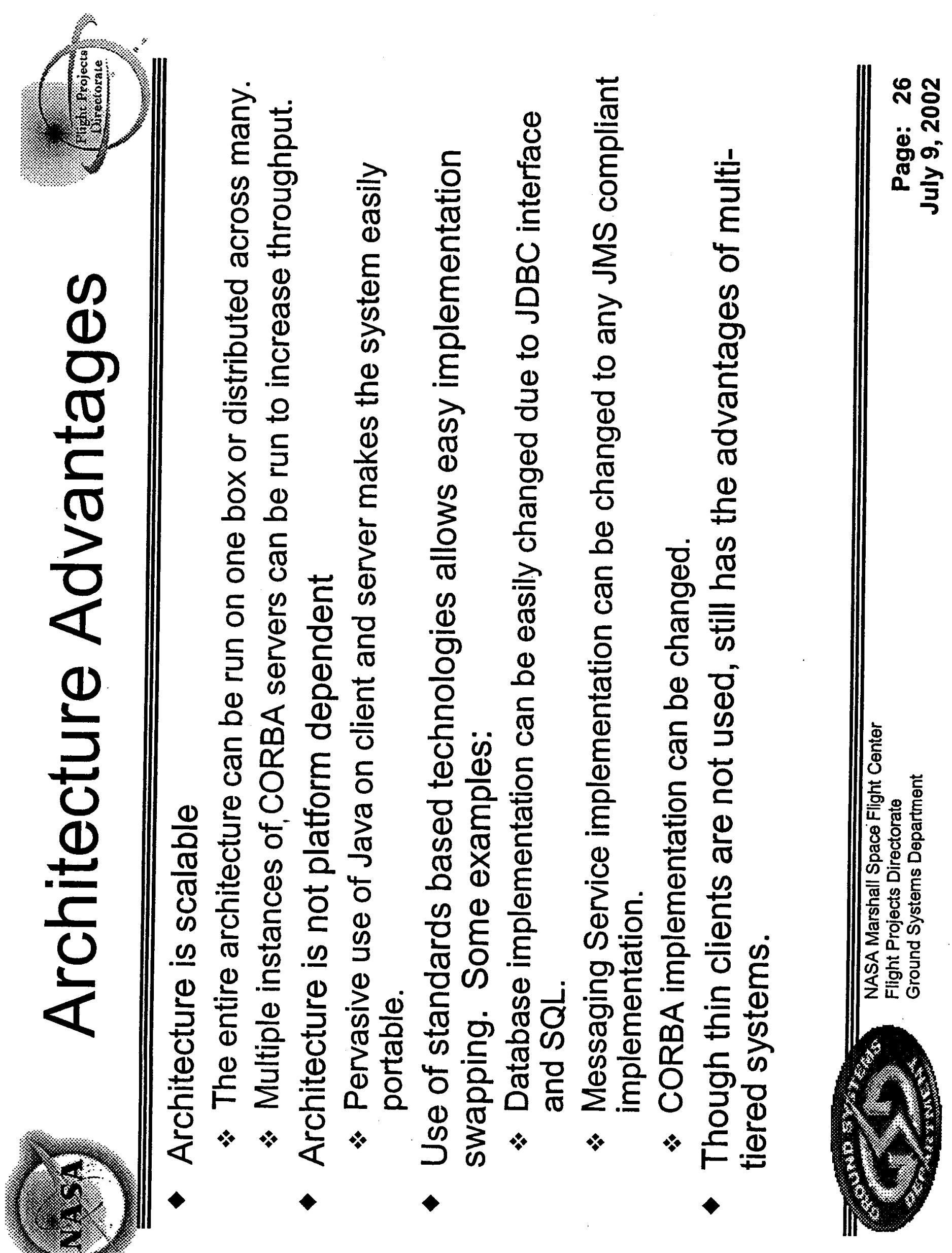




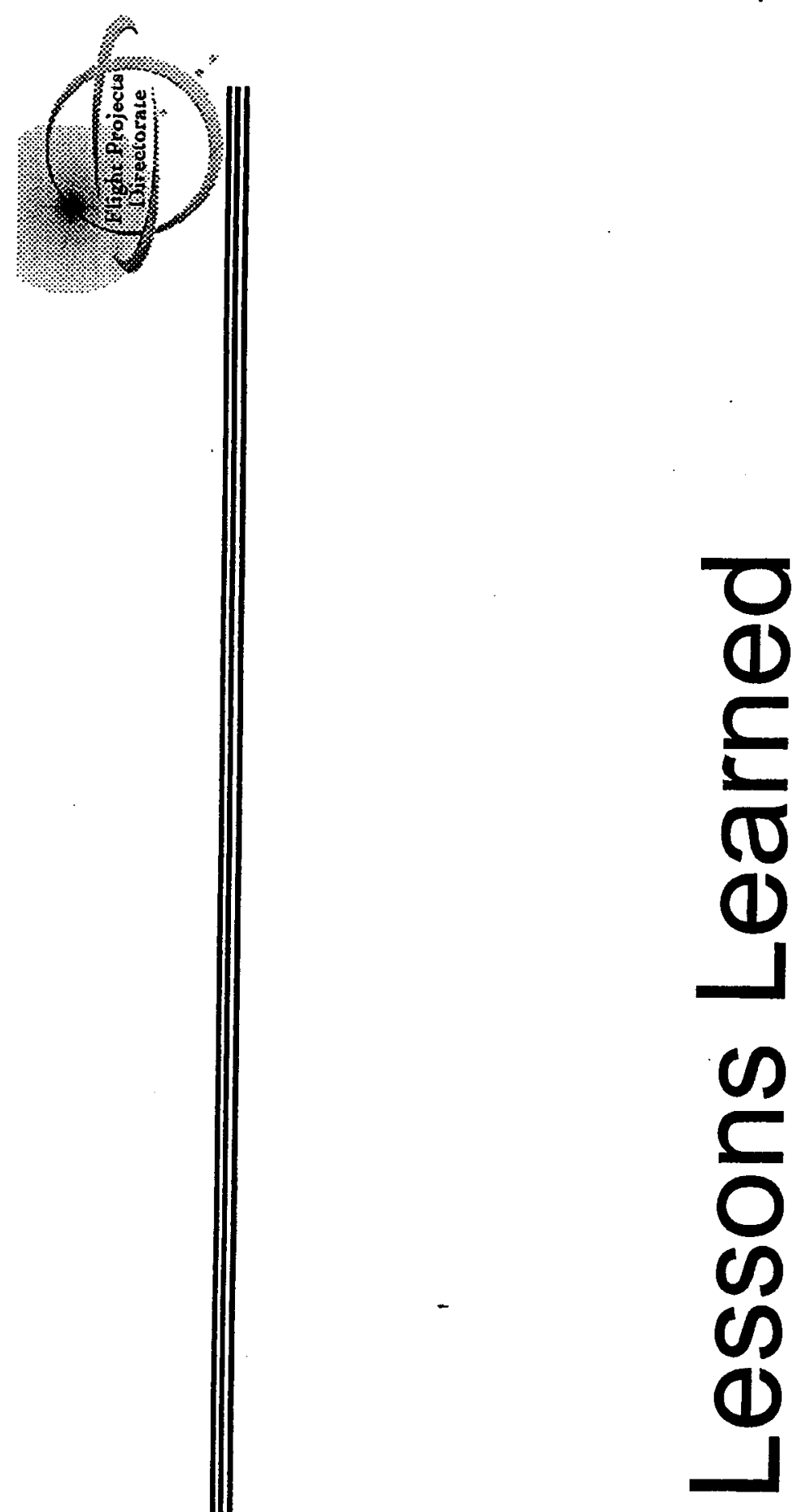

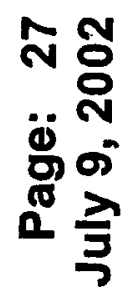

市

蒿 蒙 言要 \& 흔 응 응 の 影 容兽 西尔

这家

原空 乏立包
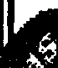

, 


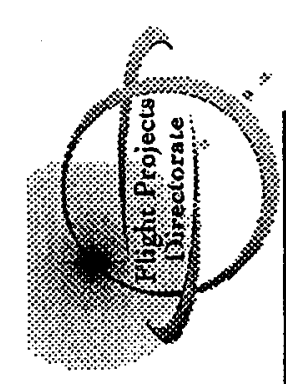

(1)

$\frac{7}{0}$

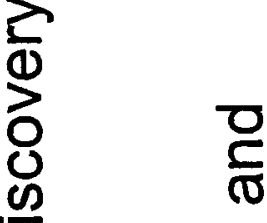

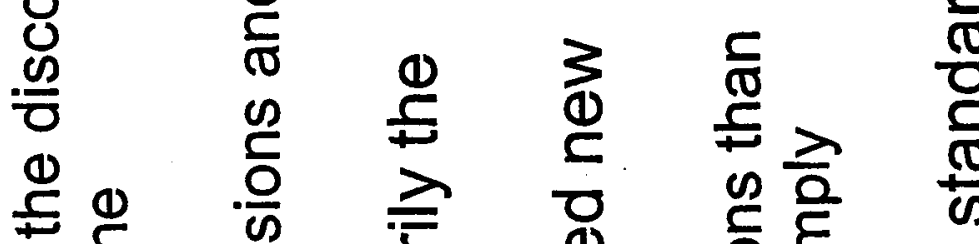

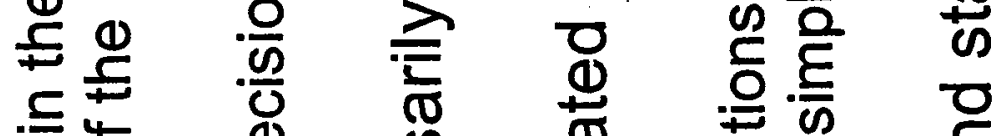

$\stackrel{0}{0}$

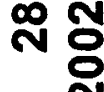

iे

1. $\frac{7}{3}$

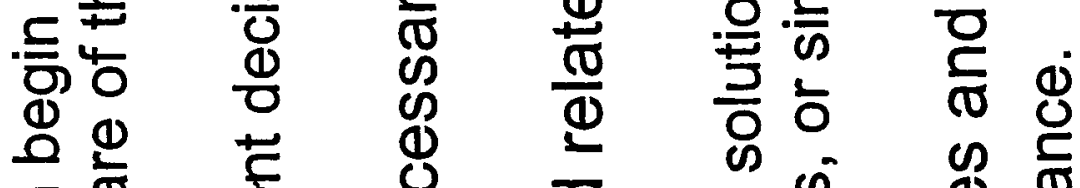

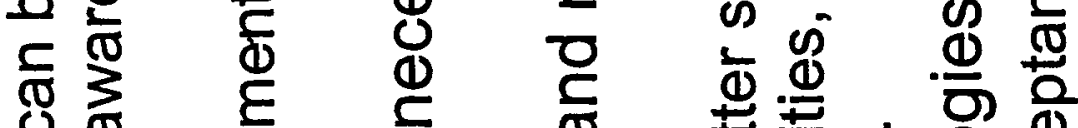

ง

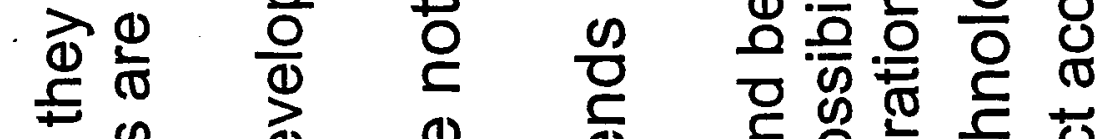

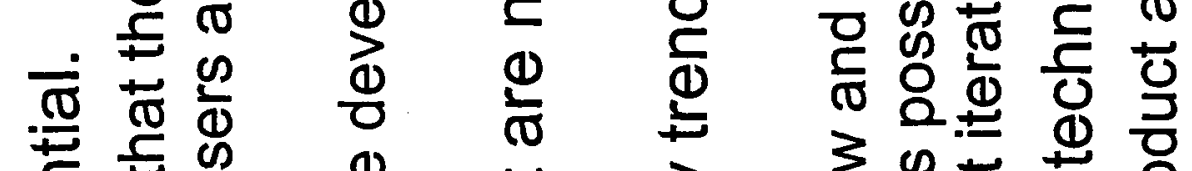

芒岳出

क

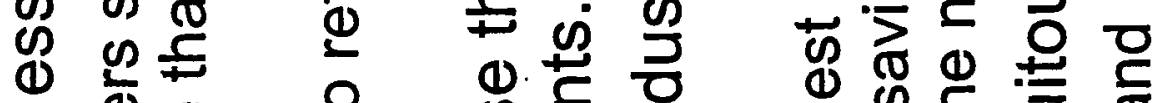

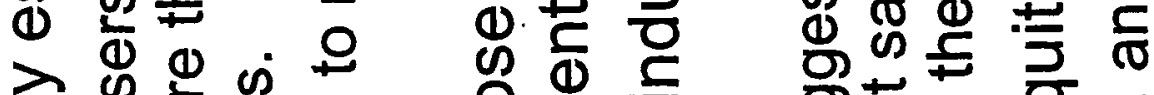

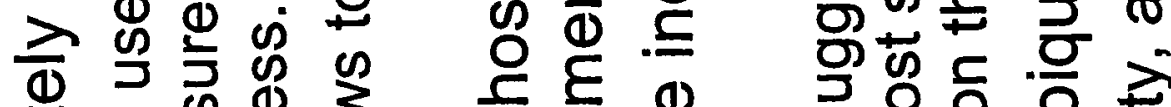

0 (1)

C

0 只

(2)

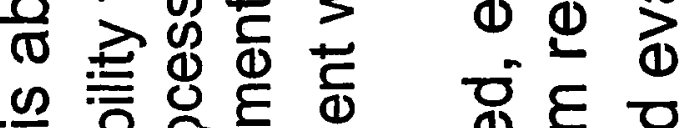

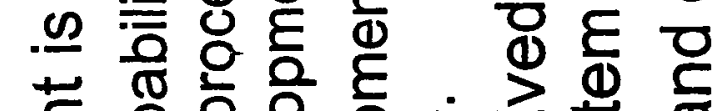

(1)

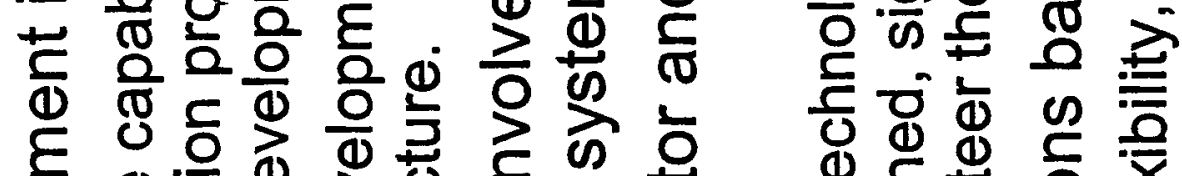

터은

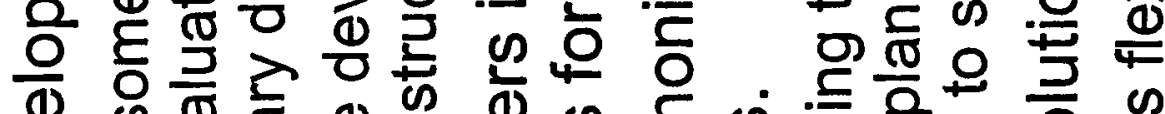

\

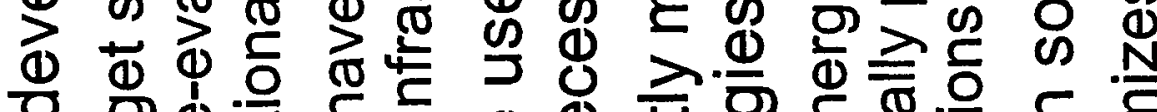

잉 d을 도

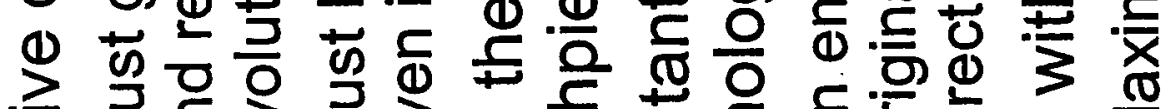

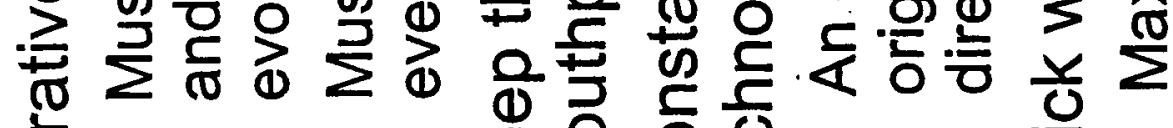

*

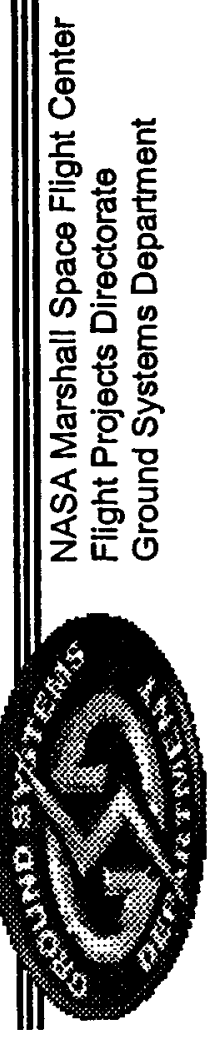




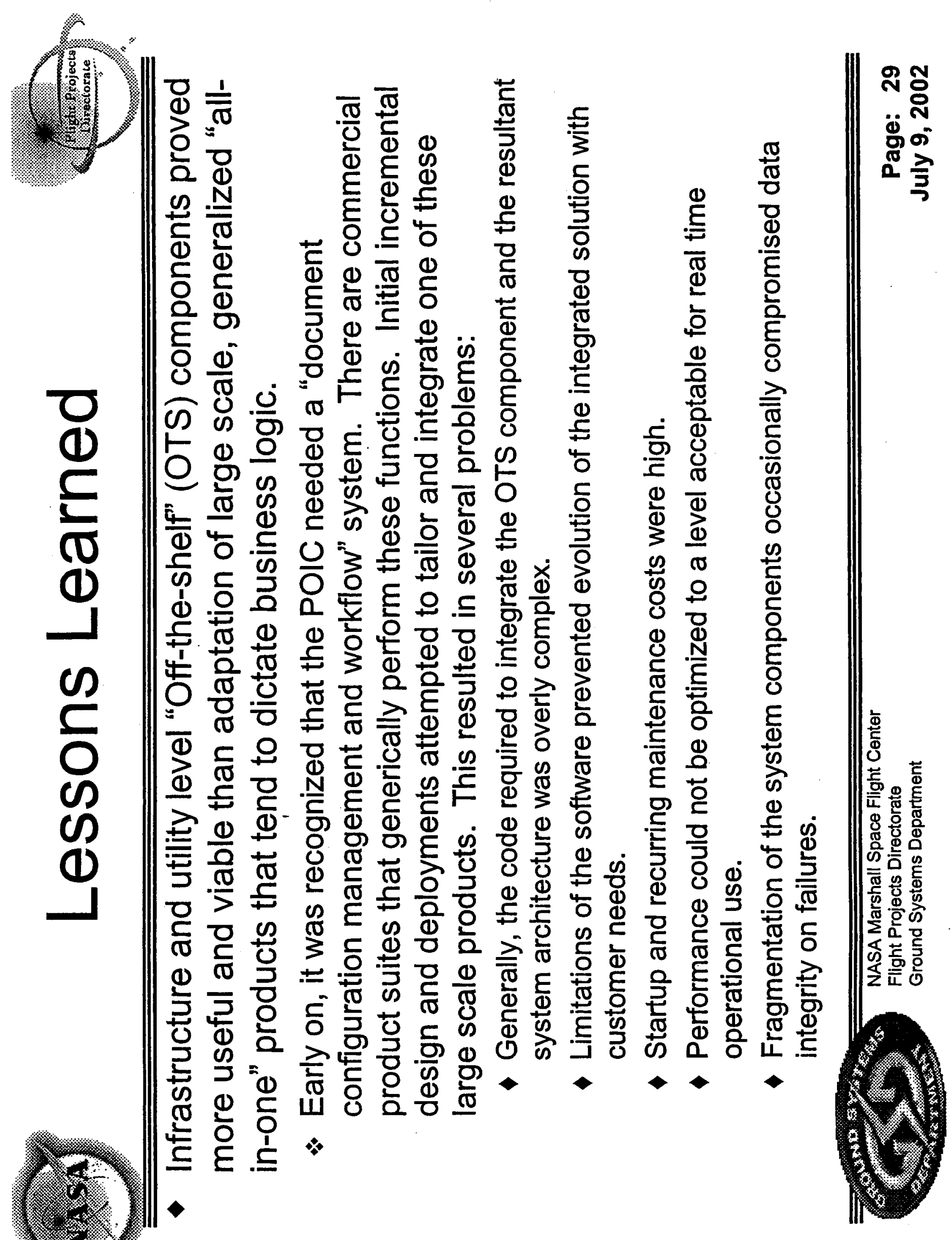




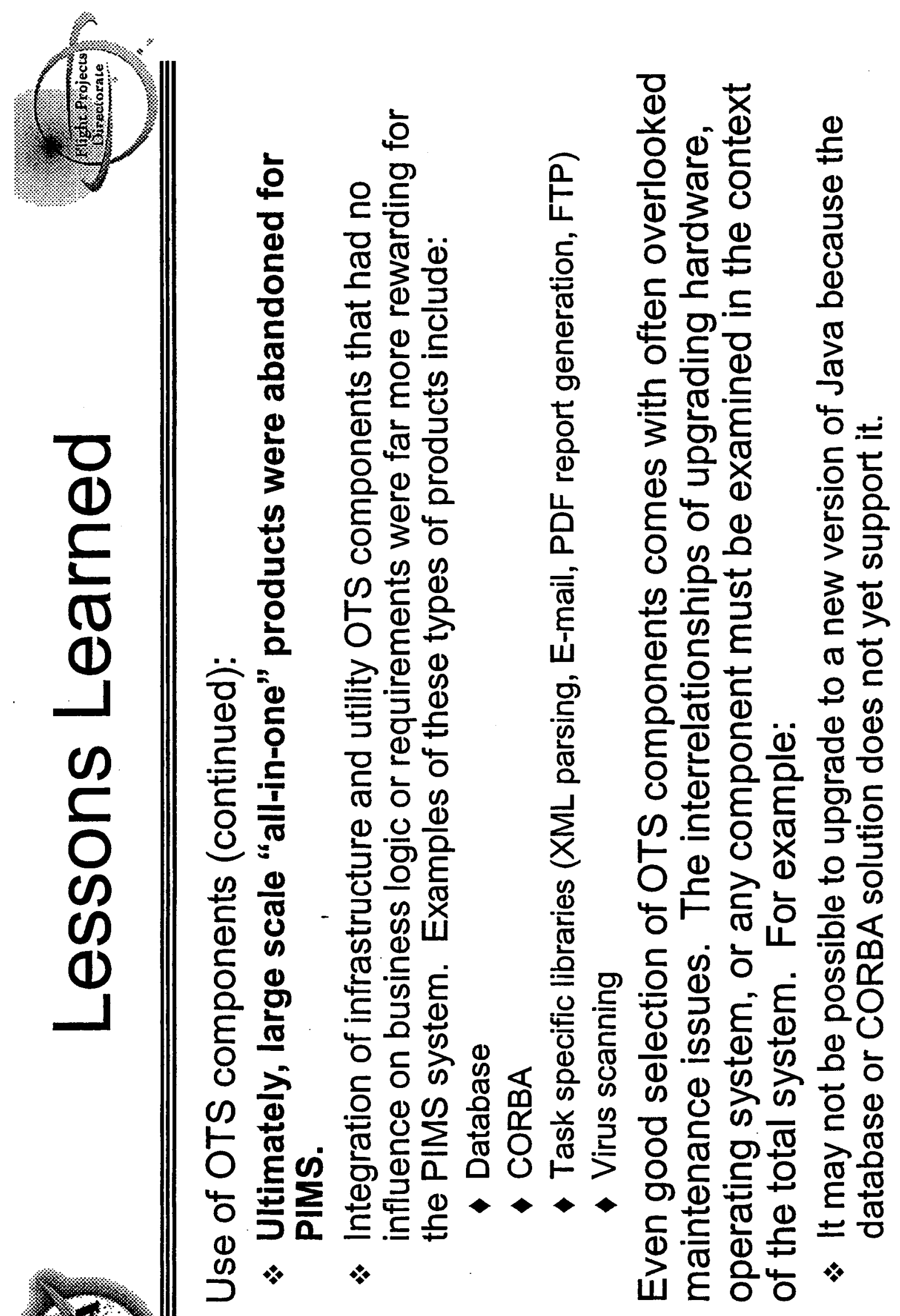




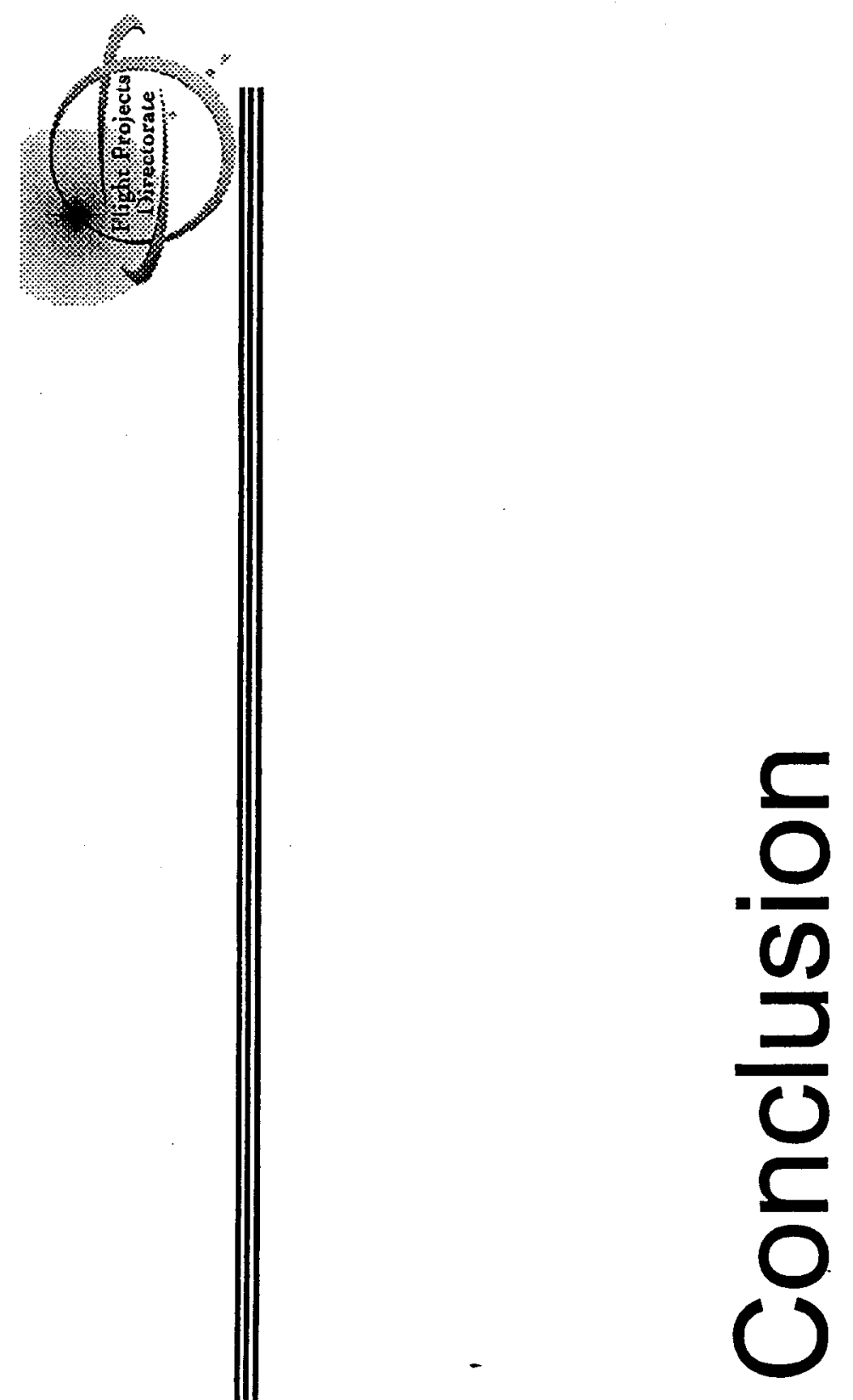

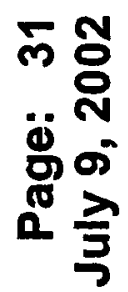

\$.

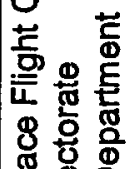

吹

क⿱ 口)

के

बั

这

安突

之竐过

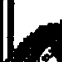




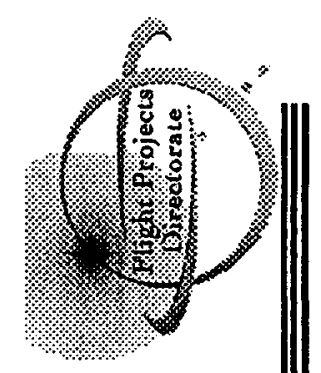

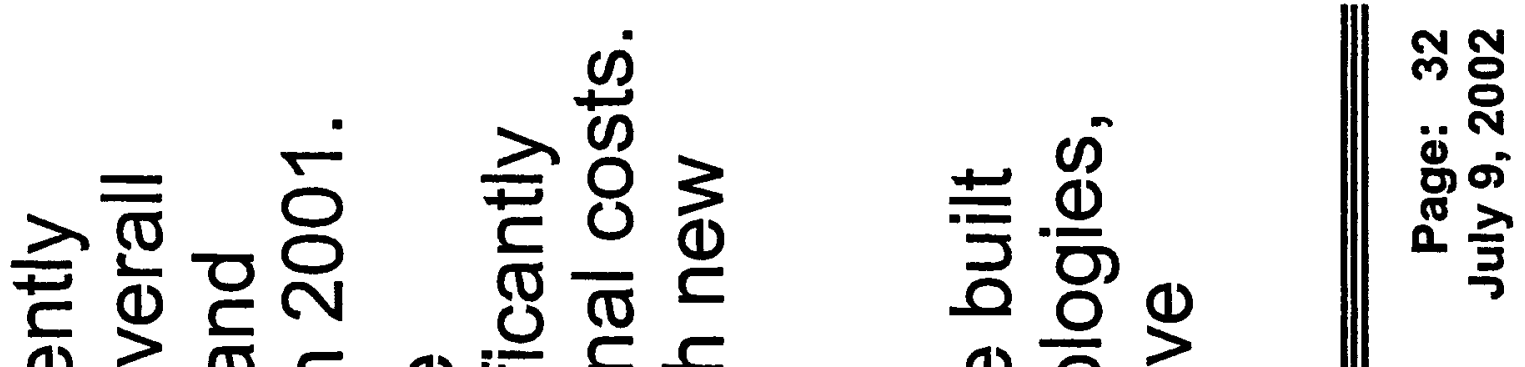

$$
\begin{aligned}
& \text { ᄃ के क⿺辶 } \\
& \text { - } \\
& \text { 以 } \\
& \text { D } \\
& \text { 응응 }
\end{aligned}
$$

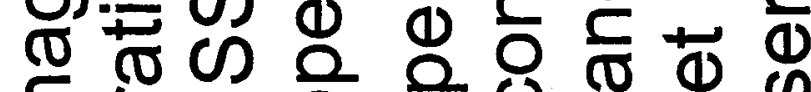

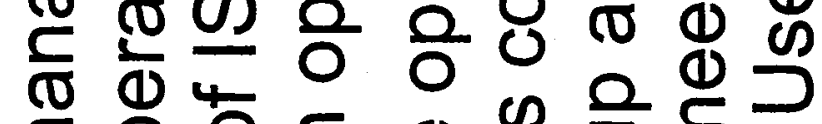

$$
\begin{aligned}
& \text { ᄃิ }
\end{aligned}
$$

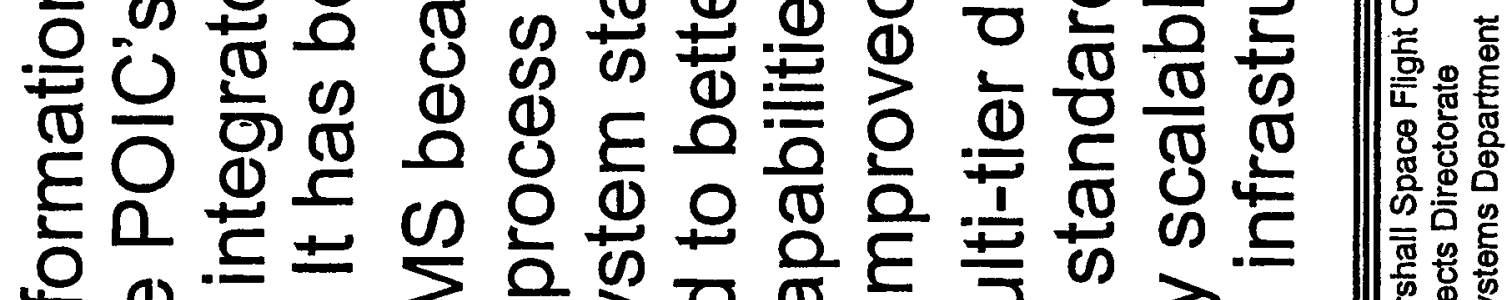

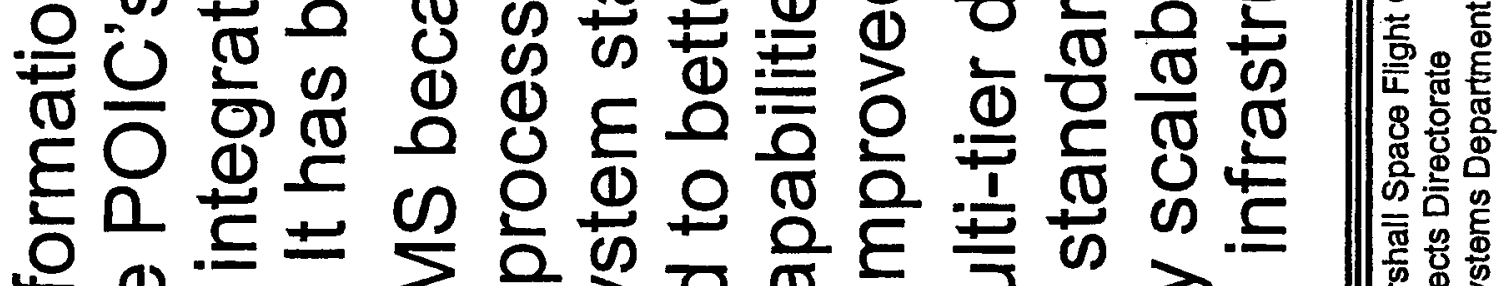

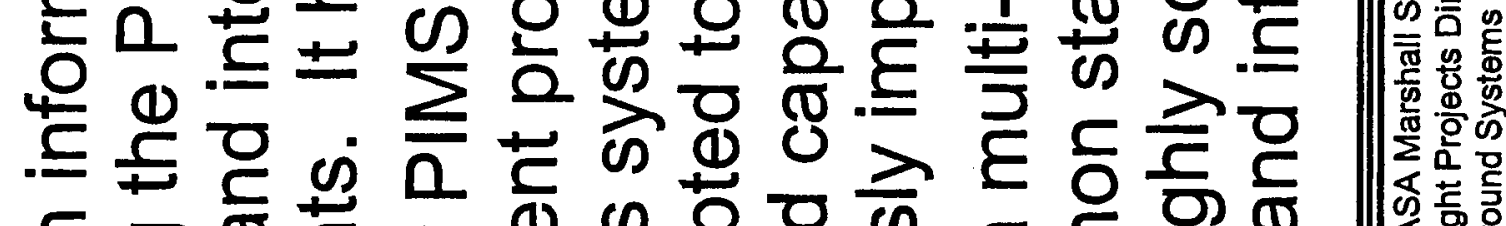

$$
\begin{aligned}
& \text { ธัธ }
\end{aligned}
$$

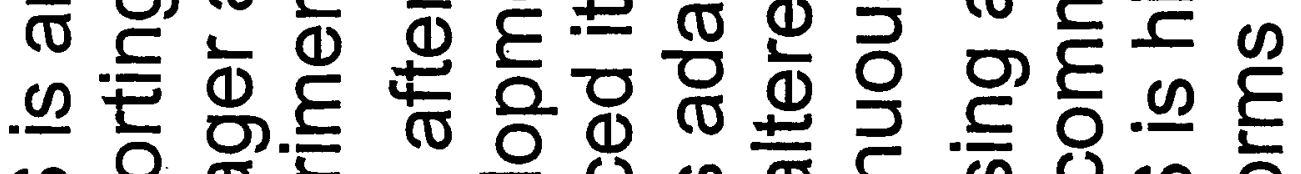

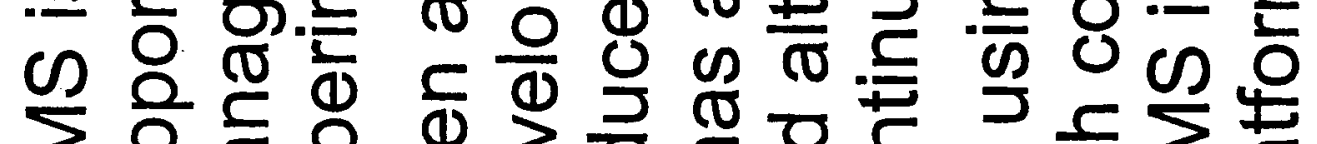

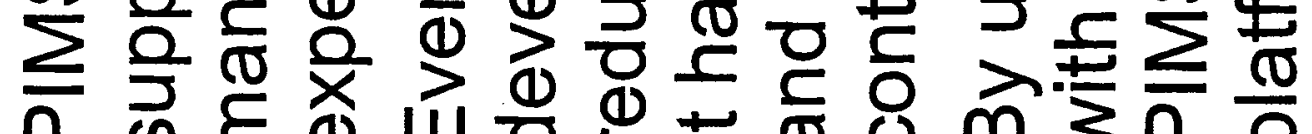




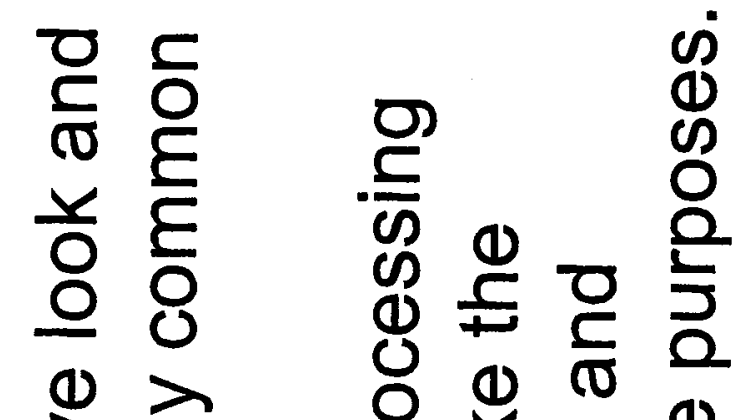

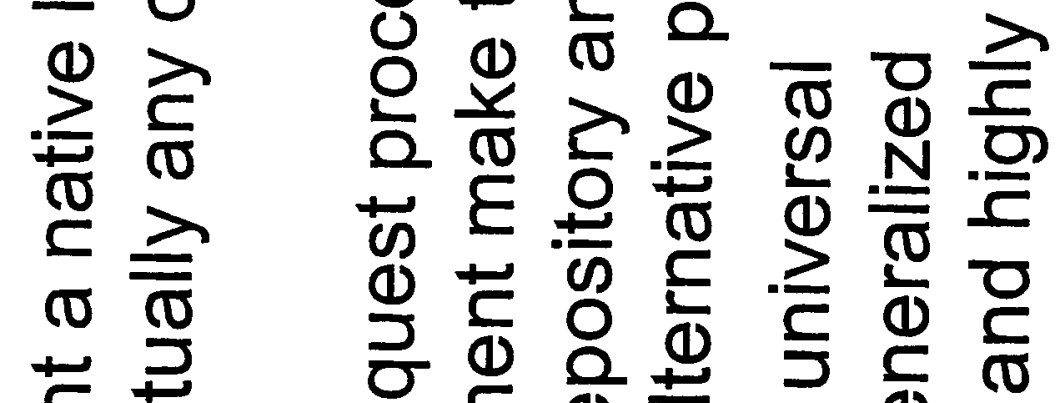

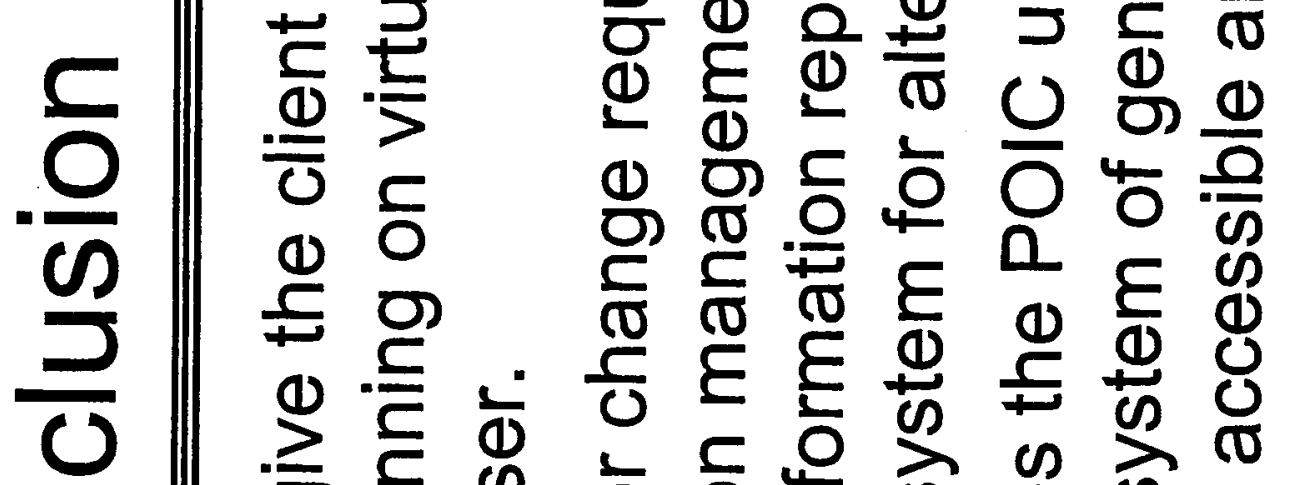

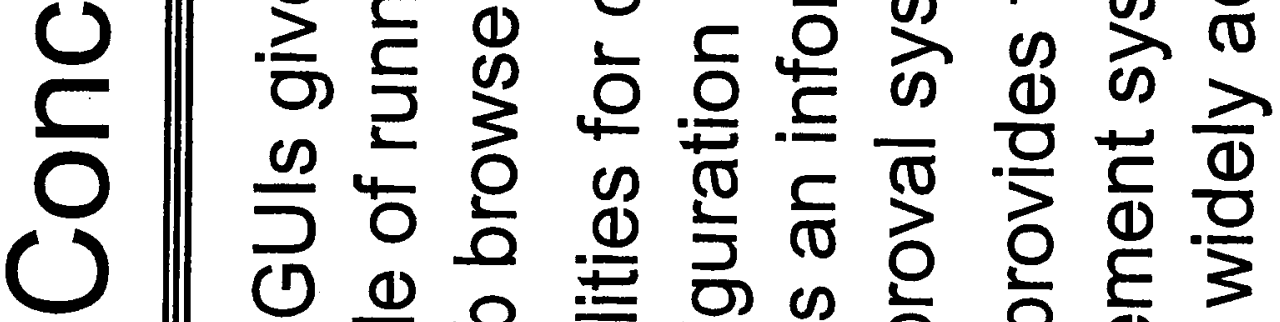

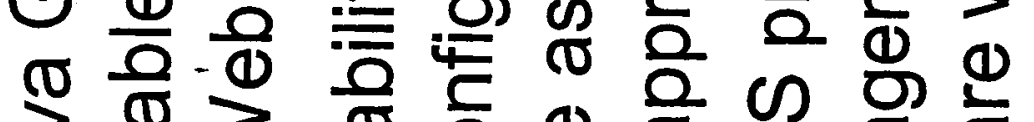

$$
\begin{aligned}
& \text { त } \\
& \supset \text { ত } \\
& \text { T E } 0 \text { 䒕 }
\end{aligned}
$$

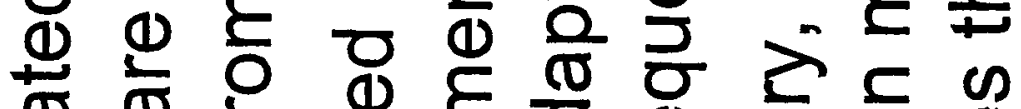

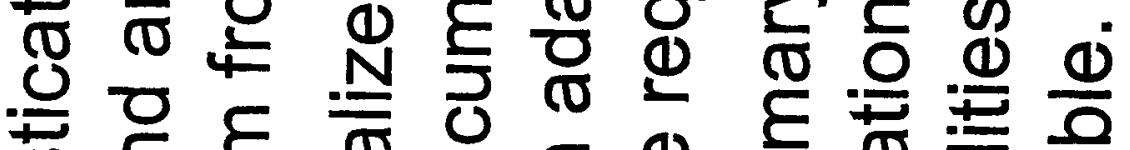

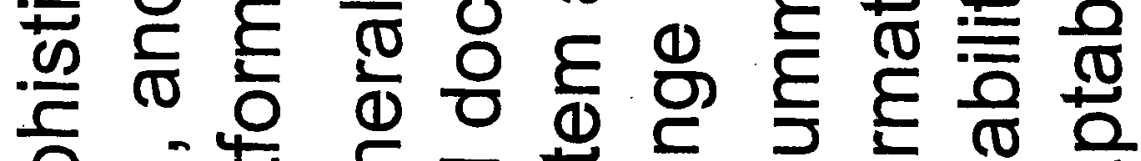

$$
\begin{aligned}
& \text { ○ंग }
\end{aligned}
$$

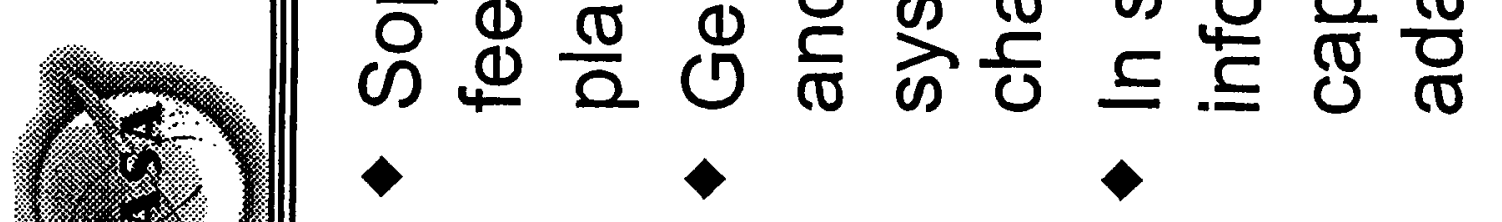

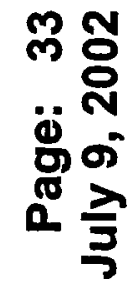

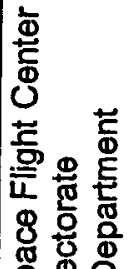$$
\text { क : }
$$$$
\text { 졷용 }
$$$$
\text { के के }
$$$$
\sum \text { 可 }
$$$$
\text { 否空 }
$$$$
\text { 之立 }
$$

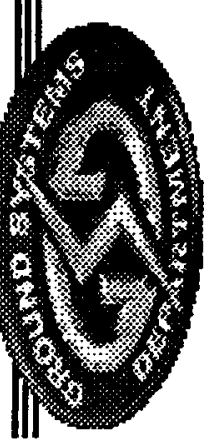



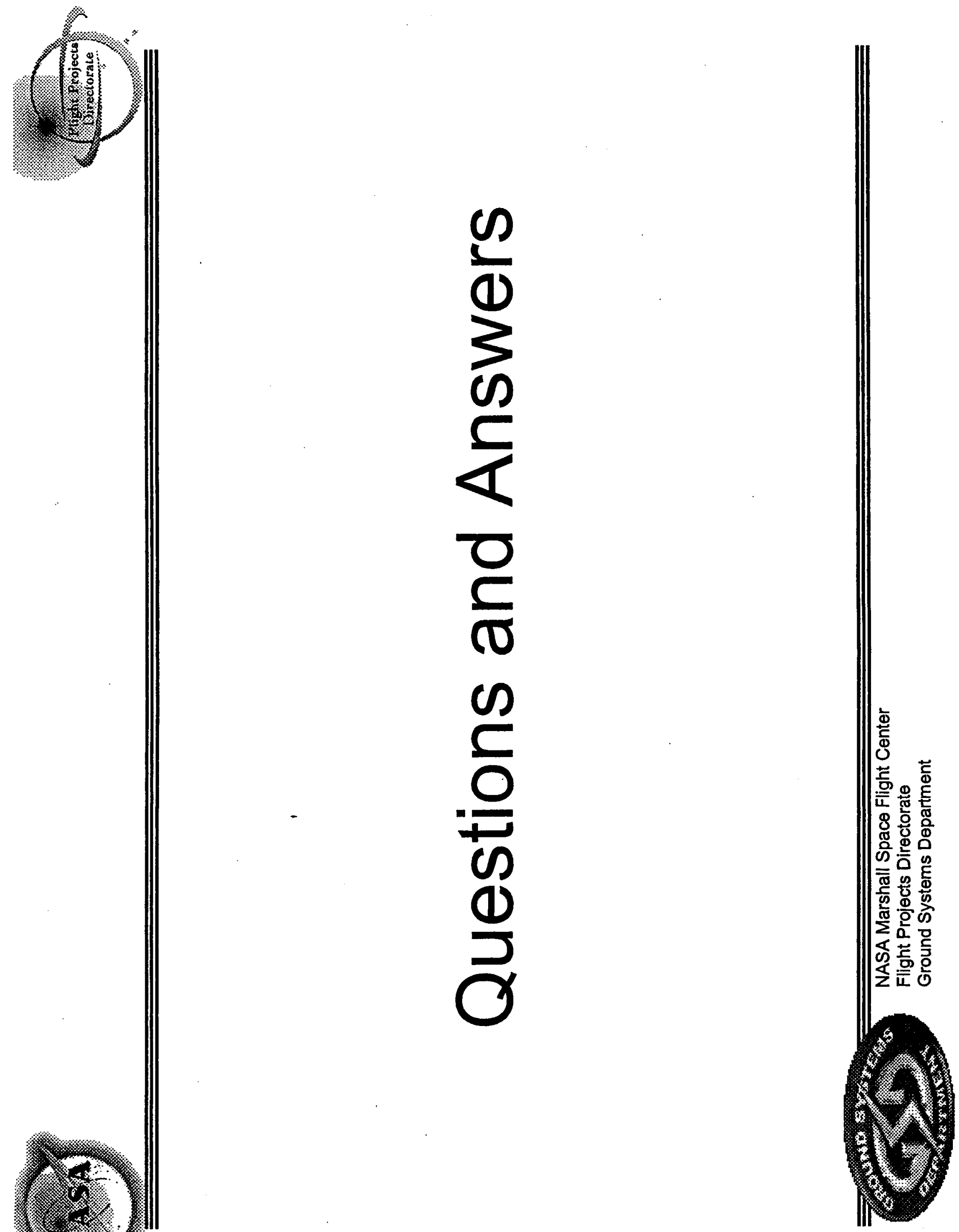